\title{
ORDENAMIENTO ECOLÓGICO GENERAL DEL TERRITORIO MEXICANO: ENFOQUE METODOLÓGICO Y PRINCIPALES EXPERIENCIAS
}

\author{
José Ramón Hernández-Santana', \\ Manuel Bollo-Manent ${ }^{2}$ \\ Ana Patricia Méndez-Linares ${ }^{1}$ \\ ${ }^{1}$ Instituto de Geografía, Universidad Nacional Autónoma de México \\ santana@igg.unam.mx, patyml@igg.unam.mx \\ ${ }^{2}$ Centro de Investigaciones en Geografía Ambiental, Universidad Nacional Autónoma de México \\ manent@gmail.com
}

\section{RESUMEN}

El presente trabajo presenta los resultados del programa de ordenamiento ecológico general del territorio mexicano (caracterización, diagnóstico, pronóstico y formulación del modelo de ocupación sectorial). Para el análisis territorial y la evaluación del estado medioambiental actual, se empleó la regionalización ambiental biofísica de López-Blanco (2008), integrada por 145 regiones. El pronóstico contempló el análisis de conflictos y sinergias intersectoriales, la realización de talleres de consulta y validación, y la construcción de los escenarios contextual (2008) y tendenciales (2012, 2023, 2033). El modelo de ordenamiento ecológico territorial incluyó 5 niveles de atención prioritaria, 80 regiones ecológicas, 34 tipos de actividades rectoras del desarrollo, 10 lineamientos ambientales generales, 44 estrategias ecológicas y 273 acciones de implementación.

Palabras clave: ordenamiento ecológico, planeación territorial, México.

\section{ABSTRACT}

The paper presents results of the Mexican environmental land use management program (description, diagnosis, forecast and formulation of sectorial occupation model). Lopez-

Fecha de recepción: diciembre 2011.

Fecha de aceptación: diciembre 2012. 
Blanco's (2008) biophysical environmental regionalization was used for the territorial analysis and evaluation of current environmental conditions, comprising 145 regions. The forecast included intersectorial conflicts and synergies analysis, consultation and validation workshops, and the creation of contextual (2008) and tendential (2012, 2023, 2033) scenarios. The environmental land use management model included 5 levels of priority attention, 80 ecological regions, 34 types of development activities, 10 general environmental guidelines, 44 ecological strategies and 273 implementation actions.

Key words: ecological planning, territorial planning, Mexico.

\section{INTRODUCCIÓN}

Las acciones cardinales para el ordenamiento territorial de cualquier espacio geográfico, sea continental, insular o marino, demandan la caracterización general y el diagnóstico de sus recursos naturales y socioeconómicos, para establecer análisis comparativos con investigaciones similares en el pasado y conocer las tendencias de su desarrollo o degradación actual. A su vez, este enfoque requiere la creación de una línea base para establecer el análisis de viabilidad ecológica de la utilización futura del territorio, ante la demanda creciente de uso por los programas y planes sectoriales gubernamentales. En este sentido, el ordenamiento ecológico general del territorio mexicano requiere de un modelo de ocupación, basado en la disponibilidad cuantitativa y en el estado cualitativo de los componentes del medio biofísico y los intereses sectoriales de la Administración Pública Federal de México (APF), que sirva de plataforma sustentable para la implementación de las políticas sectoriales socioeconómicas, con el fin de lograr la debida compatibilidad o complementariedad con las políticas ambientales requeridas en el país.

Muchos especialistas convergen, tal y como define Franco (2009), en que las acciones del ordenamiento territorial deben corresponderse con una política visionaria del Estado y representar una herramienta de planificación de los gobiernos, con objetivos primordiales de situar estratégicamente el marco de referencia espacial nacional, mediante condiciones favorables, armónicas y acordadas en consenso democrático, para mejorar la estructura socio-territorial del país, con énfasis en los asentamientos humanos, la disposición de las acciones productivas, las exigencias del desarrollo económico, y la preservación, protección y/o restauración de los recursos propios con excelencia ambiental.

En México, el ordenamiento ecológico es un instrumento de política ambiental diseñado para caracterizar, diagnosticar y proponer formas de utilización de los territorios y de sus recursos naturales, siempre bajo el enfoque del uso racional y diversificado, y con el consenso de la población (SEMARNAP, 2000). Esta necesidad de alcanzar un equilibrio dinámico entre la disponibilidad de los recursos naturales con las expectativas de vida de la población y con el desarrollo de los intereses de los diferentes sectores sociales y económicos del país y, además, con la capacidad de autorrecuperación o restauración del medio biofísico, está amparada jurídica y administrativamente, en el caso mexicano, en el reglamento de la Ley General del Equilibrio Ecológico y la Protección al Ambiente - LGEEPA (Diario Oficial de la Federación, 1988; SEMARNAT,2003), donde se establece que la SEMARNAT (Secretaría de Medio Ambiente y Recursos Naturales) está facultada para formular el Pro- 
grama de Ordenamiento Ecológico General del Territorio (POEGT), en el marco del Sistema de Planeación Democrática, contando con el consenso participativo de la sociedad y siendo un programa de observancia obligatoria en todo el territorio nacional. Asimismo, trata de vincular las acciones y programas de la APF, observando la viabilidad ambiental en términos de la Ley de Planeación.

Esta ley sustenta, que el proceso de ordenamiento ecológico territorial contempla diferentes etapas: (I) Caracterización del sistema territorial; (II) Diagnóstico integrado del sistema territorial; (III) Pronóstico, de debate público participativo; (IV) Proposición del Modelo de Ordenamiento Ecológico General del Territorio (MOEGT); y (V) Instrumentación o Implementación del mismo. Como se desprende del proceso, para conciliar las aptitudes, prioridades y necesidades de los usos del suelo, se emplea el ordenamiento ecológico del territorio, el cual se define jurídicamente como «el instrumento de política ambiental cuyo objeto es regular o inducir el uso del suelo y las actividades productivas, con el fin de lograr la protección del medio ambiente; y la preservación y el aprovechamiento sustentable de los recursos naturales, a partir del análisis de las tendencias de deterioro y las potencialidades de aprovechamiento de los mismos» (LGEEPA, Titulo Primero, Art. 3, fracción XXIII); además de reflejar, por su esencia democrática, las expectativas del desarrollo social, lo que amplia, según Fernández (2008), la cultura territorial de la población. La propia filosofía del ordenamiento territorial demanda, desde su marco teórico e incluso el legal, la implicación de la ciudadanía desde los momentos iniciales de la formulación de los planes y programas de carácter territorial, urbanístico y ambiental (Fernández, 2008), y sería totalmente antidemocrático y desastrosamente impráctico, planificar sin una eficiente comunicación ciudadana (Grabow et al., 2006).

En síntesis, este instrumento tiene como objetivo esencial, minimizar los conflictos ambientales derivados del uso del territorio y de sus recursos naturales, a través de una correcta y equilibrada planificación territorial. Al gobierno federal de México, a través de la SEMARNAT, le correspondió establecer las bases para que las Secretarías de Estado, con acciones en el territorio, tuviesen el sustento necesario para elaborar e instrumentar sus programas, con base en la aptitud territorial y las tendencias de deterioro de los recursos naturales, en los servicios ambientales, en los riesgos ocasionados por peligros naturales o tecnológicos y en la conservación del patrimonio natural. Según la propia LGEEPA, el POEGT tiene por objeto llevar a cabo una regionalización ecológica, identificando áreas de atención prioritaria y de aptitud sectorial/natural, y establecer lineamientos y estrategias ecológicas para cada una de las regiones identificadas.

En este sentido, el objetivo general del presente trabajo es presentar los principales resultados de la caracterización y diagnóstico del sistema territorial mexicano; de la evaluación de su medio ambiente bajo diferentes escenarios temporales y tendenciales, dentro de la etapa III, prospectiva o de pronóstico; y de la formulación del modelo de ocupación de los distintos programas y planes de desarrollo sectorial, en lo que representa la etapa IV, de propuesta del MOEGT, con referencia en las estrategias, lineamientos y acciones ambientales para cada una de las proposiciones sectoriales de uso territorial.

El POEGT fue financiado por las autoridades de la SEMARNAT y del Instituto Nacional de Ecología (INE), y ejecutado por un equipo de 36 investigadores y técnicos del Instituto de Geografía, del Centro de Investigaciones en Geografía Ambiental y del Centro 
Regional de Investigaciones Multidisciplinarias de la Universidad Nacional Autónoma de México (UNAM), así como por especialistas y consultores de la Universidad Autónoma de Querétaro, focalizados estos últimos, en la evaluación de las políticas públicas (Ongay y Zorrilla, 2008a) y de los requerimientos ambientales para la aptitud sectorial de cada uno de los sectores del gobierno federal de México (Ongay y Zorrilla, 2008b).

El territorio mexicano posee una extensión aproximada de $1964375 \mathrm{~km}^{2}$ (INEGI, 2008), con una rica diversidad biológica del $12 \%$ mundial (CONABIO, 2008), habitado actualmente por una población aproximada de 112322757 personas (INEGI, 2010), y representando la decimocuarta economía mundial, de acuerdo con el volumen neto de su producto interno bruto (IMF, 2009). Para el desarrollo de las investigaciones dentro del POEGT, se adoptaron como unidades de análisis territorial, a las 145 unidades ambientales biofísicas (López-Blanco, 2007, 2008), definidas por su constitución geológica, tipo de relieve, dominio morfoclimático, tipos de suelo y de vegetación, y el uso del suelo (Fig. 1).

\section{ANTECEDENTES}

El ordenamiento territorial ha derivado, en los últimos lustros, como una de las principales líneas de acción para la sustentabilidad en la gran mayoría de las naciones, representando además, el marco de referencia imprescindible para la implementación local del desarrollo sustentable (Delgado, 2006); y constituye el eje central de las estrategias de cooperación para

Figura 1

REGIONES AMBIENTALES BIOFÍSICAS DE MÉXICO (LÓPEZ-BLANCO, 2007, 2008)

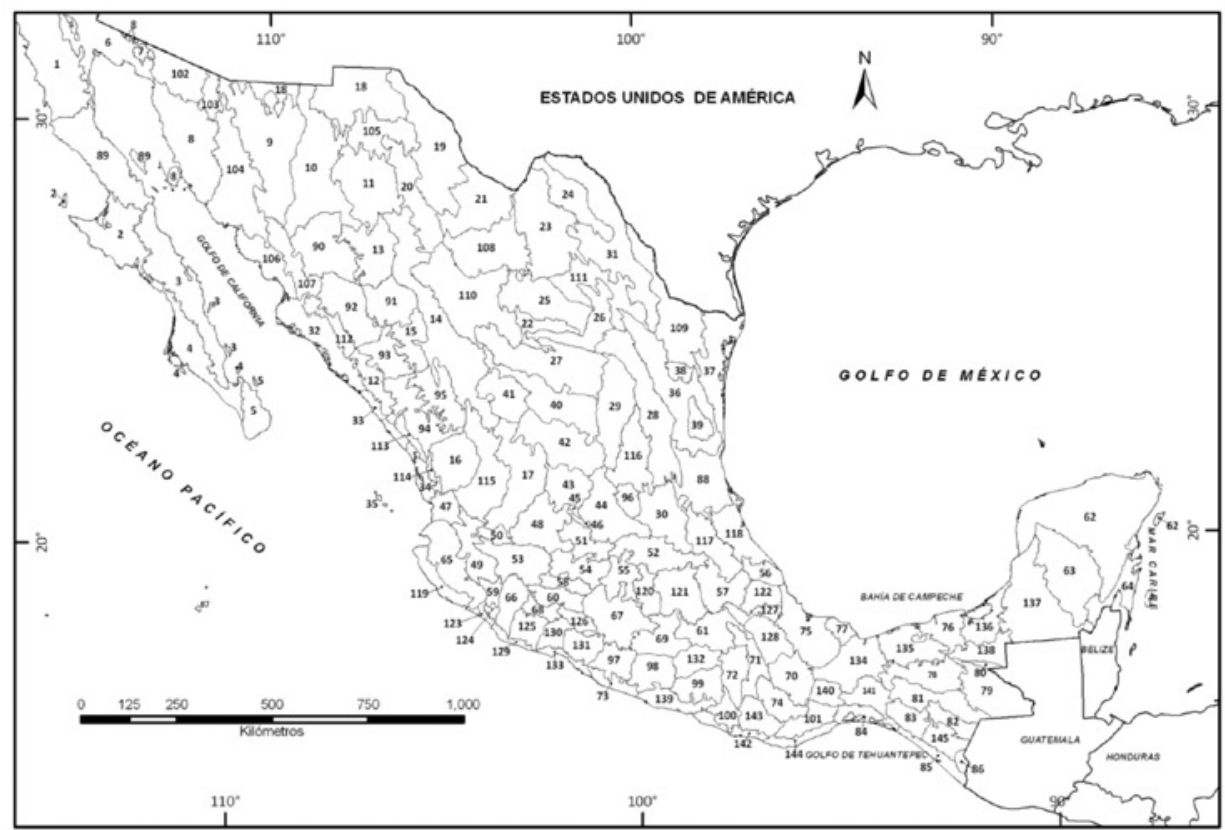


el desarrollo territorial (Romero y Farinós, 2004). Según Zoido (1998), la ordenación del territorio es esencialmente una función pública, una política compleja de reciente y escasa implantación, que puede y debe apoyarse sobre instrumentos jurídicos, sobre prácticas administrativas y en conocimientos científicos pluridisciplinarios; siendo el marco idóneo para la incorporación del paradigma ambiental en los programas de desarrollo territorial (Cuesta, 2006). En este sentido, muchos países han precisado en el enfoque ecológico del ordenamiento territorial, como ocurre en el caso de México, Colombia, Brasil, España y muchos otros países, existiendo un papel creciente de la planificación ecorregional en las políticas y estrategias de conservación y protección de la naturaleza (De Lucio et al., 2003; Gurrutxaga y Peio, 2009).

En México, durante el sexenio 1989-1994, se establece el primer intento de elaboración del POEGT. En esa oportunidad, el esfuerzo estuvo limitado por una débil participación sectorial, inexistente coordinación con el sector ambiental y carencia de información sintética y cartográfica en formato digital para el procesamiento automatizado. Más tarde, en el año 2003, se publicó el reglamento de la LGEEPA en materia de ordenamiento ecológico (SEMARNAT, 2003), pero los esfuerzos por el programa nacional no pudieron concretarse, por falta de un acuerdo y una visión intersectoriales. Finalmente, a partir del año 2006, se presentaron las condiciones de madurez metodológica, jurídica y sectorial para ejecutar el programa de ordenamiento y formular un modelo de ocupación, que orientara las políticas sectoriales de la APF, de acuerdo con las políticas ambientales requeridas en cada región. Los resultados básicos alcanzados en dicho proceso, primero de su tipo culminado en el país, se esbozan en el presente trabajo.

El POEGT de México fue decretado y publicado en el Diario Oficial de la Federación, el siete de octubre de 2012 (SEMARNAT, 2012).

\section{ENFOQUE METODOLÓGICO}

El proceso de ordenamiento ecológico general del territorio mexicano inició con la caracterización y el diagnóstico del medio biofísico nacional, a partir del conocimiento científico existente sobre el mismo y el estado actual de sus recursos, sin nueva generación de información primaria. En este sentido, se estableció la secuencia metodológica, de acuerdo con las etapas del proceso (Fig. 2).

\section{Caracterización y diagnóstico}

De acuerdo con el carácter ecológico del POEGT, uno de los primeros pasos consistió en la formulación de los productos básicos para la evaluación del estado del medio ambiente mexicano, entre ellos, la identificación de las regiones del país más vulnerables a determinados procesos y fenómenos naturales peligrosos (vulcanismo, sismicidad, huracanes, remoción en masa y variaciones del régimen hidrotérmico); el reconocimiento de las condiciones, estados, procesos y fenómenos naturales del territorio nacional, sobre todo con implicaciones históricas de degradación, como el decremento de la calidad y la cantidad del agua, la degradación de los suelos, el deterioro de la vegetación y la desertificación; el establecimiento de una regionalización ambiental (biofísica) nacional (145 
unidades ambientales biofísicas - UAB), como soporte para el análisis territorial; y la proposición de las regiones con necesidad inmediata de protección, de conservación y de restauración ambientales.

Paralelamente a este análisis del medio biofísico nacional, se establecieron las bases para la determinación de la aptitud sectorial del territorio, según atributos ambientales y criterios de cada sector de la APF, así como, la identificación de las áreas de conflictos y sinergias intersectoriales, en talleres regionales con la participación de representantes de los diferentes sectores, a niveles federal y estatales. Ambos insumos contribuyeron a la definición de los niveles de atención prioritaria para cada una de las unidades ambientales biofísicas y a la fundamentación de la regionalización ecológica (ecólogo-productiva) decretada en la LGEEPA y desarrollada durante la etapa de pronóstico.

\section{Pronóstico}

Para el desarrollo de una visión prospectiva, a diferentes plazos, fue indispensable el empleo y la ponderación de un conjunto de indicadores que respondieran a la definición holística de medio ambiente (Bucek, 1983; Arcia, 1994; Mateo et al., 2001) y que posibilitaran la creación del escenario contextual del año 2008 y de las tendencias temporales (años 2012, 2023 y 2033), así como, el escenario estratégico, de implementación territorial de políticas ambientales, a partir del análisis integrado del estado actual del medio ambiente de cada una de las 145 UAB y del nivel de conflictos y/o sinergias intersectoriales en cada una de las mismas.

Para la evaluación del estado actual del medio ambiente, se aplicó la teoría de la evaluación geoecológica (Mateo et al., 1994), con el empleo de 18 indicadores de degradación biofísica, modificación antrópica, situación socioeconómica y disponibilidad de recursos hídricos superficiales y subterráneos (Tabla 1). La evaluación de cada uno de estos indicadores por unidades ambientales biofísicas, aparece como versión reducida en la Tabla 2. Las evaluaciones de pronóstico para los años 2012, 2023 y 2033 (Tabla 3), realizadas por los autores, se discutieron y avalaron en talleres regionales desarrollados en las ciudades de Guadalajara, Distrito Federal y Tuxtla Gutiérrez, con la intervención de los diferentes sectores socio-productivos. Este trabajo se fortaleció durante el proceso final de consultas públicas nacionales, desarrollado durante los años 2010 y 2011, con la celebración, además, de talleres en las ciudades de Hermosillo, Monterrey, Guadalajara, Distrito Federal, Oaxaca y Mérida, como sedes de las grandes regiones del país, como Noroeste, Noreste, Occidente, Centro, Sur y Sureste respectivamente.

\section{Proposición del Modelo de Ordenamiento Ecológico General del Territorio (MOEGT)}

Una de las tareas primordiales para la determinación de la aptitud sectorial fue el conocimiento detallado de las políticas públicas federales y de cada sector, así como, la definición de sus atributos ambientales como sustento de las áreas de interés sectorial en el país. Esta información permitió la determinación de la aptitud sectorial para cada una de las 145 UAB, 
Figura 2

FLUJOGRAMA DEL PROCESO METODOLÓGICO DEL PROGRAMA DE ORDENAMIENTO ECOLÓGICO GENERAL DEL TERRITORIO, EN EL CASO DE MÉXICO

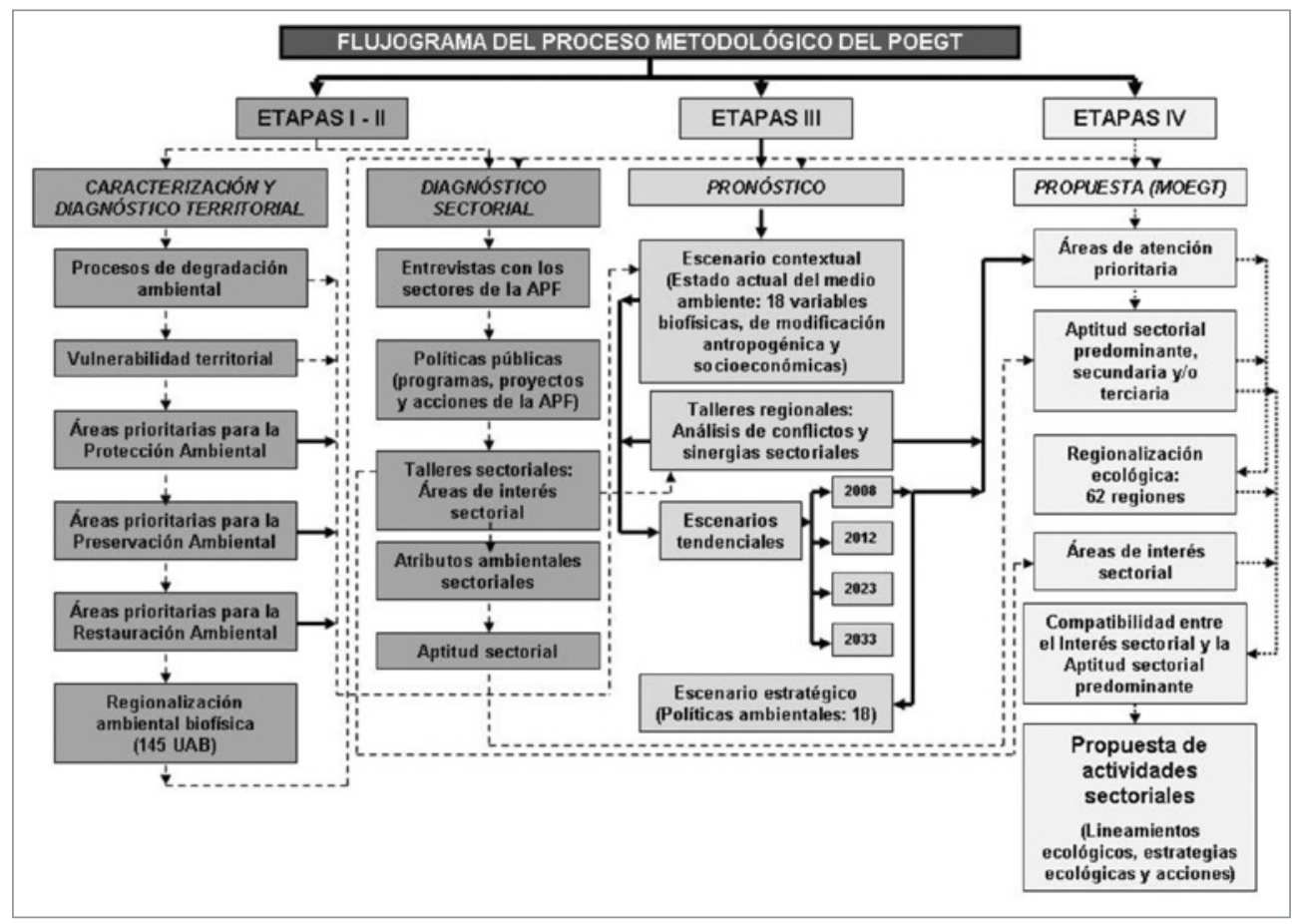

reconociendo a las UAB más aptas para cada actividad sectorial y a las que presentan intereses de ocupación por los sectores de la APF.

A partir del estado actual del medio ambiente (escenario contextual del año 2008) y del nivel de conflictos intersectoriales en cada una de las unidades ambientales biofísicas, se identificaron y clasificaron los niveles de atención prioritaria (muy alta, alta, media, baja y muy baja) de cada una de ellas (Tabla 3). Esto reveló las UAB con mayor grado de afectación ambiental y las más litigantes por la competencia intersectorial, que sin dudas incrementarán los daños al medio ambiente a futuro, algunos con carácter irreversible.

Sobre la base de la trilogía técnico-jurídica (política ambiental propuesta, nivel de atención prioritaria y aptitud sectorial), plasmada en la LGEEPA, se estableció la regionalización ecológica del territorio nacional, compuesta por 80 tipos de regiones ecólogo-productivas, que a su vez, indicaron las vocaciones óptimas de uso sectorial y la urgente necesidad de apoyo presupuestario federal para su recuperación. Para cada una de estas regiones ecológicas y sus unidades ambientales biofísicas, fue determinado el grado de compatibilidad entre su aptitud sectorial y el interés sectorial de uso, con vistas a identificar el nivel de compatibilidad con la oferta ambiental de cada unidad. Esta etapa permitió discriminar esos intereses y fomentar las demandas sectoriales compatibles. 


\begin{tabular}{|c|c|c|c|c|c|c|c|c|c|c|c|c|c|c|c|c|c|c|}
\hline & 量 & $\frac{8}{\frac{1}{\infty}}$ & $\stackrel{\circ}{\wedge}$ & $\stackrel{2}{\wedge}$ & $\stackrel{ }{\wedge}$ & $\begin{array}{l}\bar{\Xi} \\
\stackrel{\Delta}{\wedge}\end{array}$ & $\stackrel{O}{\dot{\leftrightarrow}} \stackrel{0}{\stackrel{0}{+}}$ & $\underset{\wedge}{\vec{\sigma}}$ & 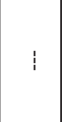 & : & 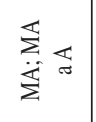 & $\begin{array}{l}\text { ڤे̀े } \\
\text { bे }\end{array}$ & לे̀े & 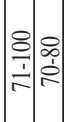 & $\begin{array}{l}\stackrel{\Xi}{0} \\
\stackrel{\circ}{\wedge} \\
\stackrel{1}{*}\end{array}$ & 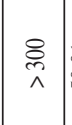 & 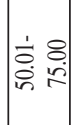 & 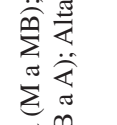 \\
\hline क & 현 & $\frac{\stackrel{D}{0}}{i b}$ & $\begin{array}{l}\stackrel{a}{a} \\
\stackrel{1}{c}\end{array}$ & $\frac{n}{\frac{n}{n}}$ & $\frac{}{ㅁ}$ & 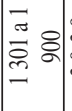 & ले. & $\underset{\stackrel{8}{9}}{\stackrel{8}{9}}$ & 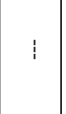 & 1 & 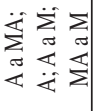 & 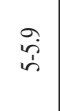 & iे & 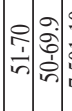 & $\begin{array}{l}\frac{0}{1} \\
\frac{1}{2} \\
\frac{2}{2}\end{array}$ & $\frac{8}{\stackrel{c}{2}}$ & 容 & 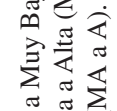 \\
\hline $\begin{array}{l}0 \\
0 \\
0 \\
0 \\
z \\
z\end{array}$ & : & 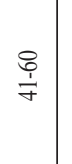 & $\begin{array}{l}\stackrel{9}{\bar{J}} \\
\dot{\infty} \\
\infty\end{array}$ & 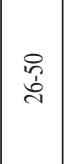 & $\stackrel{i}{i}$ & $\begin{array}{l}\stackrel{8}{0} \\
\frac{\pi}{2} \\
\overrightarrow{2}\end{array}$ & 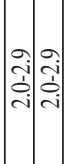 & $\frac{8}{\frac{1}{1}}$ & 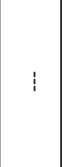 & 17 & 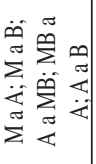 & f & $\stackrel{g}{\dot{q}}$ & 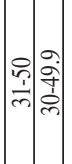 & 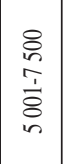 & 总 & $\begin{array}{l}8 \\
\stackrel{0}{0} \\
\stackrel{\oplus}{1} \\
\dot{0} \\
\stackrel{+}{0}\end{array}$ & 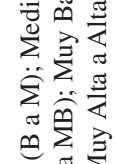 \\
\hline 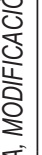 & : & $\frac{g}{i}$ & $\stackrel{9}{\stackrel{9}{+}}$ & $\begin{array}{l}\text { خे } \\
\text { o }\end{array}$ & $\vec{v}$ & 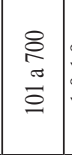 & : & in & 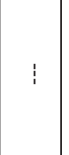 & 15 & 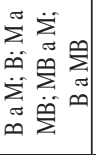 & $\vec{m}$ & ले & ๖ิ? & $\mid \begin{array}{c}8 \\
8 \\
1 \\
5 \\
0 \\
2 \\
2\end{array}$ & $\begin{array}{l}\stackrel{+}{1} \\
\frac{1}{n}\end{array}$ & $\begin{array}{l}8 \\
\stackrel{0}{1} \\
\dot{1} \\
\dot{0} \\
0\end{array}$ & 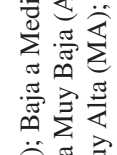 \\
\hline$\frac{0}{0}$ & 送 & స్రి & $\dot{\vec{v}}$ & $\stackrel{\circ}{v}$ & 谓 & $\frac{8}{v}$ & $\begin{array}{ll}a & \dot{a} \\
\dot{v} & \dot{v} \\
v\end{array}$ & $\frac{n}{v}$ & : & 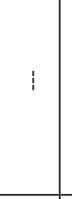 & 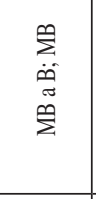 & ते & సे & $\because \frac{9}{ \pm}$ & $\mid \begin{array}{l}8 \\
\stackrel{D}{n} \\
\vec{v}\end{array}$ & $\frac{n}{1}$ & $\begin{array}{l}8 \\
0 \\
0 \\
0 \\
0 \\
0\end{array}$ & 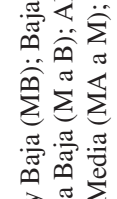 \\
\hline 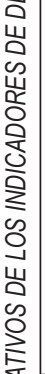 & 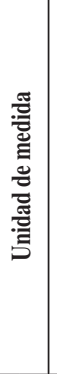 & 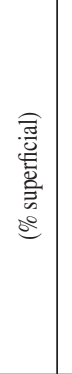 & 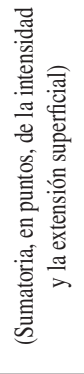 & 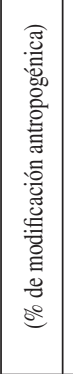 & 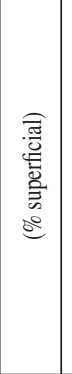 & 畺 & 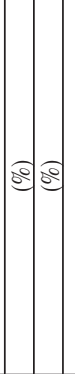 & 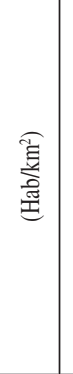 & $\stackrel{\gtrless}{z}$ & $\sum_{i}^{\circ}$ & 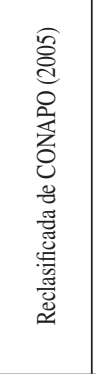 & ت & ) & : & 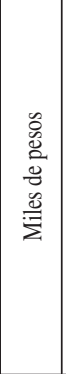 & 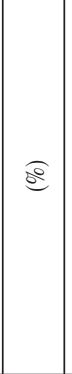 & $\approx$ & 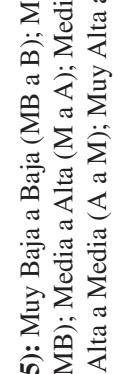 \\
\hline 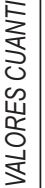 & 递 & 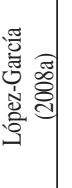 & 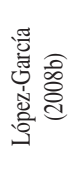 & 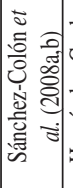 & 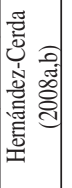 & 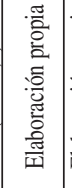 & 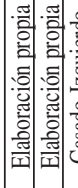 & 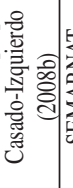 & 窟 & 帘 & 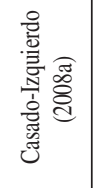 & 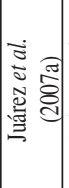 & 㲵 & 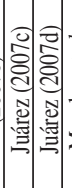 & 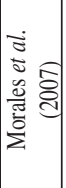 & 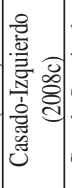 & 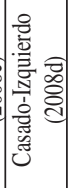 & 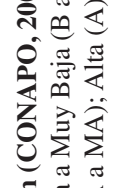 \\
\hline 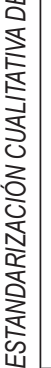 & 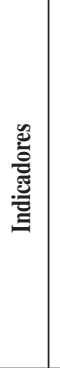 & 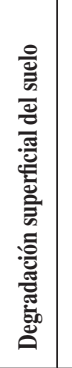 & 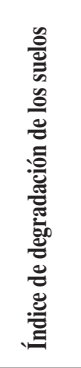 & 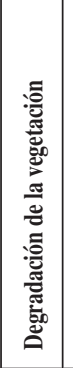 & 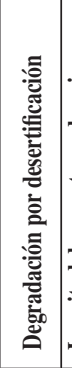 & 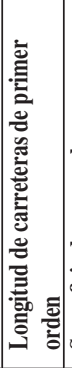 & 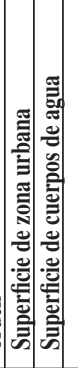 & 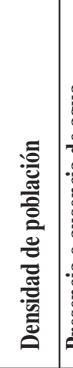 & 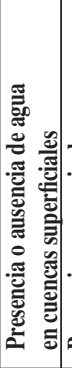 & 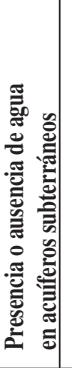 & 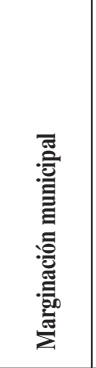 & 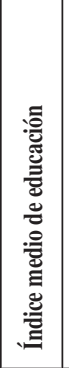 & 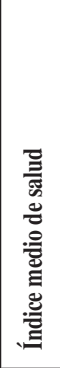 & 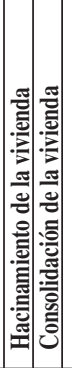 & 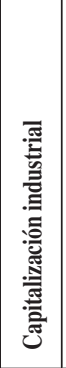 & 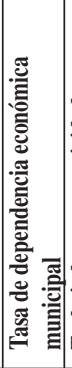 & 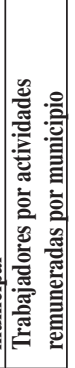 & 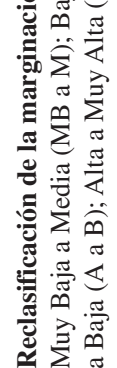 \\
\hline
\end{tabular}




\begin{tabular}{|c|c|c|c|c|c|c|c|c|c|c|c|c|c|c|c|c|c|c|c|c|}
\hline 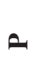 & 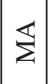 & $\ll$ & $\varangle$ & $\varangle$ & 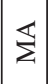 & 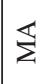 & 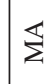 & $\mathbb{\Sigma}$ & $\ll$ & $\ll$ & $\varangle$ & 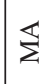 & $\Sigma$ & $\Sigma$ & $\Sigma$ & $\Sigma$ & $\varangle$ & 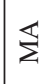 & $\mathbb{\Sigma}$ & 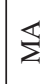 \\
\hline 0 & $\infty$ & 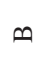 & $\infty$ & $\infty$ & $\stackrel{p}{\Sigma}$ & $\infty$ & $\infty$ & $\infty$ & $\infty$ & $\Sigma$ & $\varangle$ & $\infty$ & $\mathbb{\Sigma}$ & $\mathbb{\Sigma}$ & $\varangle$ & $\mathbb{\Sigma}$ & 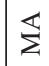 & $\Sigma$ & $\infty$ & $\infty$ \\
\hline ' & $\Sigma$ & $\stackrel{n}{\Sigma}$ & $\stackrel{p}{\Sigma}$ & $\stackrel{p}{\Sigma}$ & $\stackrel{n}{\Sigma}$ & $\Sigma$ & $\Sigma$ & $\Sigma$ & $\Sigma$ & $\Sigma$ & $\Sigma$ & $\sum_{\Sigma}^{p}$ & $\Sigma$ & $\infty$ & $\infty$ & $\stackrel{\eta}{\Sigma}$ & $\stackrel{\vartheta}{\Sigma}$ & $\stackrel{m}{\Sigma}$ & $\Sigma$ & $\Sigma$ \\
\hline $\mathbf{z}$ & $\infty$ & $\stackrel{n}{\Sigma}$ & $\stackrel{\oplus}{\Sigma}$ & $\infty$ & $\Sigma$ & $\stackrel{\rho}{\Sigma}$ & $\stackrel{\varphi}{\Sigma}$ & $\infty$ & $\sum$ & $\stackrel{n}{\Sigma}$ & $\stackrel{\oplus}{\Sigma}$ & $\Sigma$ & $\stackrel{n}{\Sigma}$ & $\stackrel{m}{\Sigma}$ & $\stackrel{\vartheta}{\Sigma}$ & $\stackrel{\eta}{\Sigma}$ & $\infty$ & $\sum$ & $\stackrel{\vartheta}{\Sigma}$ & $\infty$ \\
\hline$\Sigma$ & $\infty$ & $\infty$ & $\infty$ & $\infty$ & $\Sigma$ & $\infty$ & $\infty$ & $\infty$ & $\infty$ & $\infty$ & $\infty$ & $\Sigma$ & $\Sigma$ & $\infty$ & $\infty$ & $\varangle$ & $\infty$ & $\infty$ & $\infty$ & $A$ \\
\hline$\Xi$ & $\Sigma$ & $\ll$ & $\varangle$ & $\varangle$ & $\varangle$ & $\Sigma$ & 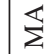 & $\Sigma$ & $\infty$ & $\infty$ & $\infty$ & $\Sigma$ & $\Sigma$ & $\Sigma$ & $\Sigma$ & $\varangle$ & $\Sigma$ & $\stackrel{p}{\Sigma}$ & $\infty$ & $\infty$ \\
\hline - & $\mathbb{\Sigma}$ & 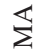 & $\mathbb{\Sigma}$ & $\varangle$ & 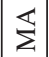 & $\mathbb{\Sigma}$ & 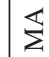 & 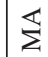 & $\Sigma$ & $\varangle$ & $\Sigma$ & $\infty$ & $\stackrel{\vartheta}{\Sigma}$ & $\Sigma$ & $\infty$ & $\stackrel{\wp}{\Sigma}$ & $\Sigma$ & $\varangle$ & $\Sigma$ & $\varangle$ \\
\hline 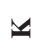 & $\stackrel{n}{\Sigma}$ & $\varphi$ & $\stackrel{\vartheta}{\Sigma}$ & $\stackrel{\rho}{\Sigma}$ & $\sum_{\Sigma}^{q}$ & $\sum$ & $\sum_{\Sigma}^{n}$ & $\stackrel{\oplus}{\Sigma}$ & $\sum$ & $\infty$ & $\infty$ & $\Sigma$ & 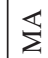 & $\infty$ & $\varangle$ & $\mathbb{\Sigma}$ & $\infty$ & $\sum$ & $\infty$ & $\stackrel{\varphi}{\Sigma}$ \\
\hline$r$ & z & z & 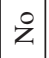 & z & $\stackrel{0}{z}$ & z̊ & $\stackrel{\circ}{z}$ & z & $\bar{n}$ & $\bar{s}$ & $z_{z}$ & $\bar{n}$ & $\bar{\sim}$ & $z$ & $\vec{w}$ & $\ddot{n}$ & ż & $\stackrel{0}{z}$ & 官 & $\ddot{z}$ \\
\hline- & $\bar{n}$ & $\bar{n}$ & $\vec{n}$ & $\bar{s}$ & $\vec{n}$ & 合 & 合 & ż & $\bar{n}$ & $\bar{v}$ & ह & $\vec{w}$ & के & $\vec{n}$ & $\vec{n}$ & $\ddot{n}$ & $\ddot{n}$ & 合 & 官 & 穴 \\
\hline $\boldsymbol{I}$ & $\Sigma$ & $\stackrel{\eta}{\Sigma}$ & $\stackrel{\vartheta}{\Sigma}$ & $\stackrel{\eta}{\Sigma}$ & $\infty$ & $\infty$ & $\stackrel{n}{\Sigma}$ & $\stackrel{\eta}{\Sigma}$ & $\stackrel{\varphi}{\Sigma}$ & $\stackrel{n}{\Sigma}$ & $\stackrel{\eta}{\Sigma}$ & $\stackrel{\vartheta}{\Sigma}$ & $\stackrel{n}{\Sigma}$ & $\stackrel{m}{\Sigma}$ & $\stackrel{\vartheta}{\Sigma}$ & $\stackrel{m}{\Sigma}$ & $\infty$ & $\sum^{\eta}$ & $\infty$ & $\Sigma$ \\
\hline ש & $\stackrel{\eta}{\Sigma}$ & $\stackrel{\eta}{\Sigma}$ & $\stackrel{m}{\Sigma}$ & $\stackrel{n}{\Sigma}$ & $\stackrel{\eta}{\Sigma}$ & $\stackrel{n}{\Sigma}$ & 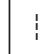 & $\stackrel{n}{\Sigma}$ & $\stackrel{\vartheta}{\Sigma}$ & $\stackrel{q}{\Sigma}$ & $\infty$ & $\infty$ & $\stackrel{\varphi}{\Sigma}$ & $\stackrel{n}{\Sigma}$ & 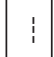 & $\stackrel{m}{\Sigma}$ & $\stackrel{n}{\Sigma}$ & $\stackrel{\eta}{\Sigma}$ & $\stackrel{m}{\Sigma}$ & $ص$ \\
\hline[ & $\infty$ & $\stackrel{\wp}{\Sigma}$ & 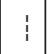 & $\frac{\eta}{\Sigma}$ & $\sum_{\Sigma}^{q}$ & $\infty$ & 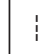 & $\stackrel{\wp}{\Sigma}$ & $\stackrel{\vartheta}{\Sigma}$ & $\vdots$ & $\stackrel{\wp}{\Sigma}$ & $\stackrel{p}{\Sigma}$ & $\stackrel{\vartheta}{\Sigma}$ & $\stackrel{m}{\Sigma}$ & $\frac{\eta}{\Sigma}$ & $\stackrel{\vartheta}{\Sigma}$ & $\stackrel{\wp}{\Sigma}$ & $\frac{\eta}{\Sigma}$ & $\stackrel{\emptyset}{\Sigma}$ & $ص$ \\
\hline [디 & $\Sigma$ & $\infty$ & $\infty$ & $\infty$ & $\infty$ & $\Sigma$ & $\sum_{\Sigma}^{n}$ & $\Sigma$ & $\Sigma$ & $\infty$ & $\Sigma$ & $\infty$ & $\infty$ & $\varangle$ & $\stackrel{\eta}{\Sigma}$ & $\stackrel{p}{\Sigma}$ & $\Sigma$ & $\Sigma$ & $\infty$ & $\Sigma$ \\
\hline O & $\infty$ & 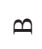 & $\infty$ & $\infty$ & $\infty$ & : & 寻 & $\Sigma$ & $\Sigma$ & $\infty$ & $\infty$ & 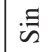 & $\Sigma$ & $\Sigma$ & $\stackrel{\Xi}{n}$ & 政 & $\Sigma$ & $\mathbb{\Sigma}$ & $\Sigma$ & $\Sigma$ \\
\hline U & $\infty$ & : & $\approx$ & $\infty$ & $: \Xi$ & $\Sigma$ & 品 & $\infty$ & $\infty$ & $\infty$ & $\Sigma$ & $\Sigma$ & $\Sigma$ & $\varangle$ & $\Sigma$ & $\varangle$ & $\varangle$ & 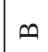 & : & $\Sigma$ \\
\hline 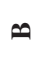 & $\infty$ & $\sum^{1}$ & $\stackrel{\vartheta}{\Sigma}$ & $\stackrel{\eta}{\Sigma}$ & $\stackrel{\varphi}{\Sigma}$ & $\infty$ & $\stackrel{\varphi}{\Sigma}$ & $\varangle$ & $\infty$ & $\stackrel{n}{\Sigma}$ & $\varangle$ & $\infty$ & $\varangle$ & $\Sigma$ & $\Sigma$ & $\Sigma$ & $\varangle$ & $\Sigma$ & $\varangle$ & $\varphi$ \\
\hline$\varangle$ & $\vec{z}$ & ? & 学 & 学 & $\begin{array}{l}z_{1} \\
0 \\
0 \\
1\end{array}$ & 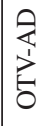 & ? & t & $\mid \begin{array}{l}0 \\
0 \\
\frac{1}{1} \\
\lambda^{\prime} \\
0\end{array}$ & $\Psi$ & $\underset{\substack{1\\
}}{0}$ & 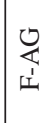 & $\begin{array}{l}\vec{z} \\
0 \\
0 \\
\text { 工1 }\end{array}$ & 茛 & L & $\Phi$ & 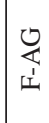 & z & 忞 & 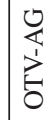 \\
\hline$\approx$ & $1-$ & $\sim$ & $m$ & $\theta$ & $n$ & 6 & $r$ & $\infty$ & $a$ & $\varrho$ & $\Xi$ & $\simeq$ & $\stackrel{m}{2}$ & \pm & $\because$ & 0 & $=$ & $\stackrel{\infty}{\sim}$ & 2 & ¿ి \\
\hline
\end{tabular}

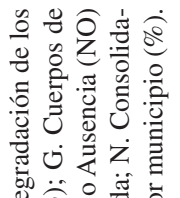

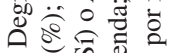

$\varphi$

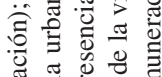

要

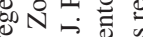

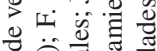

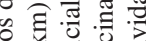

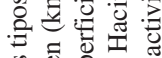

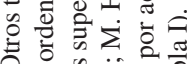

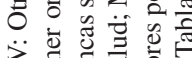

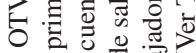

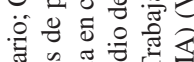

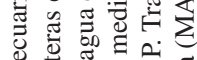

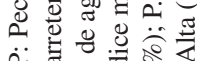

․ ฮु

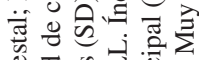

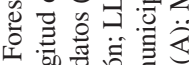

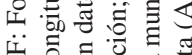

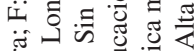

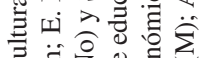

芒艺导

政

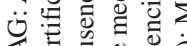

远完吾

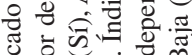

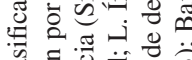

洁

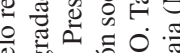

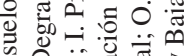

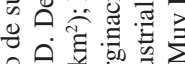

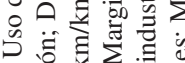

\& 氜

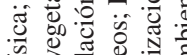

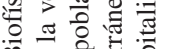

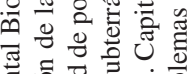

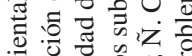

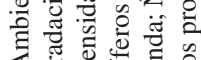

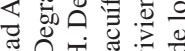

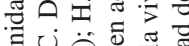

5 o $\approx 0$

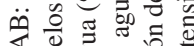




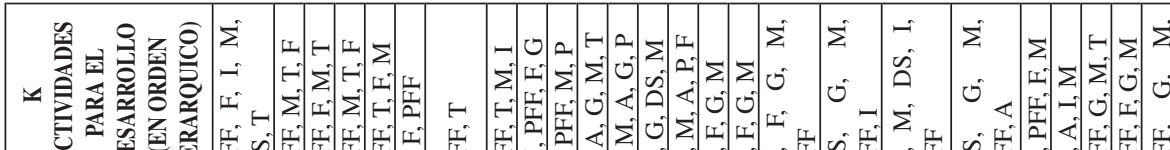

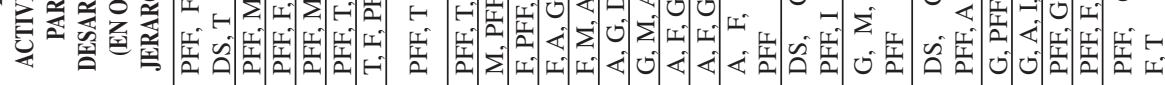

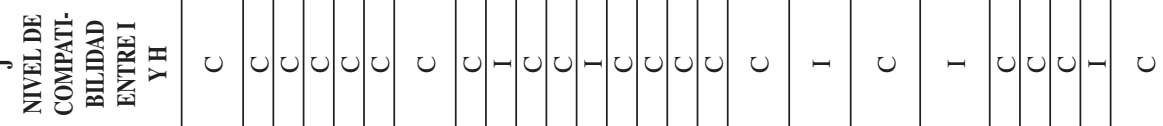

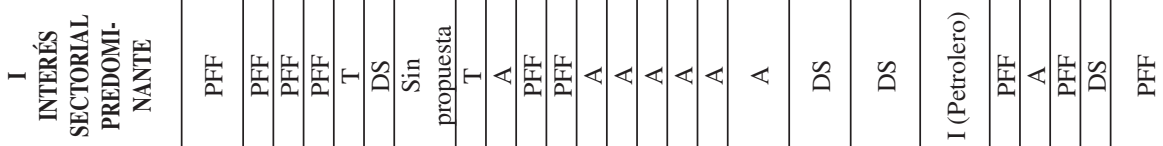

ড়

崖

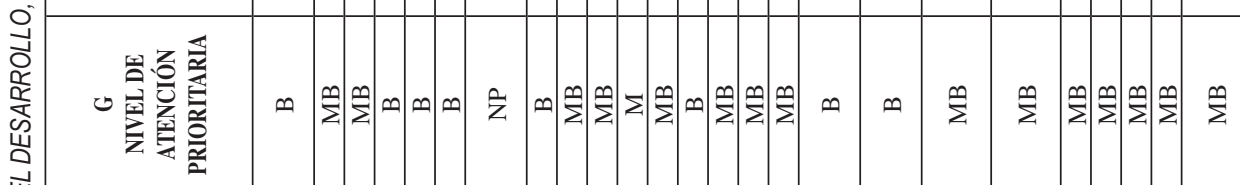

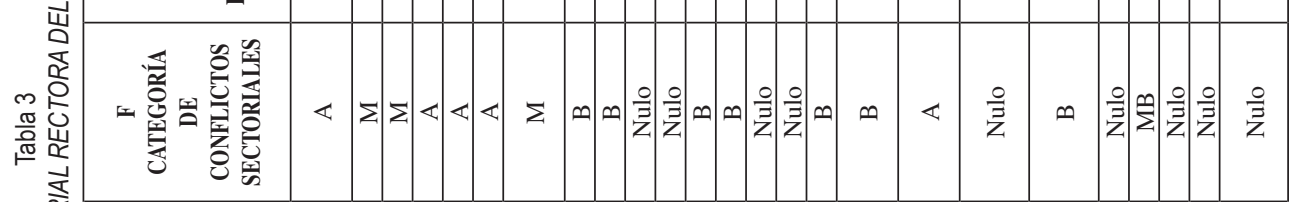

\begin{tabular}{|c|c|c|c|c|c|c|c|c|c|c|c|c|c|c|c|c|c|}
\hline 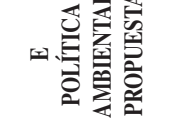 & 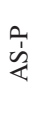 & 2 & : & & $\vec{z}$ & 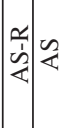 & 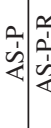 & $\frac{1}{4}<\frac{x}{4}$ & $<\frac{2}{4}$ & $\mid \begin{array}{lll}1 \\
2\end{array}$ & $\stackrel{\text { qu }}{2}$ & $\begin{array}{l}\frac{1}{1} \\
\frac{1}{4}\end{array}$ & 装 & \& & $\mid \begin{array}{ll}0 \\
\vdots \\
2\end{array}$ & 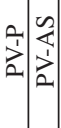 & \\
\hline 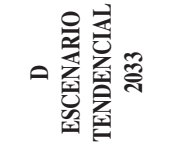 & 苇 & 兼 & 苾 & 范芫 & 并 & 己 & נُ & 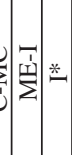 & $\approx *$ & 关 & - & 艺 & 呪 & 莡 & 羍 & $\stackrel{*}{*} \stackrel{*}{*}^{*}$ & \\
\hline 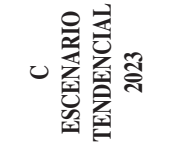 & 㸒 & 恶 & & $\stackrel{n}{n}$ & $\stackrel{T}{\underline{1}}$ & 证 & | & $\sum^{n}=1$ & $\sum \mid \vec{z}$ & 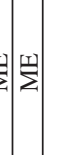 & $\stackrel{5}{\sum^{1}}$ & - & $\stackrel{n}{\Sigma}$ & - & $\omega \mid$ & 떼 띠 & \\
\hline 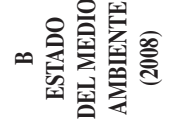 & 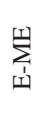 & $\sum_{1}^{1}$ & & 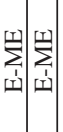 & $\sum_{1}^{1}$ & 住 & - & $-\frac{1}{2}$ & $\sum \sum$ & $\vec{a}$ & 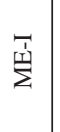 & $\sum_{\Sigma}^{1}$ & $\sum_{i}^{+}$ & $\sum_{\Sigma}^{\amalg}$ & $\omega \mid \sum_{\omega}^{N}$ & 피 & \\
\hline 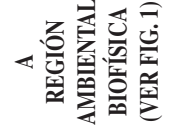 & - & $\sim \rho$ & & in 0 & $r$ & $\infty \sigma$ & $=$ & $\Rightarrow \cong \cong$ & $\pm \bumpeq$ & $0=$ & $\simeq$ & $\stackrel{\infty}{-}$ & $\stackrel{2}{2}$ & సి & $1-$ & તેป & \\
\hline
\end{tabular}




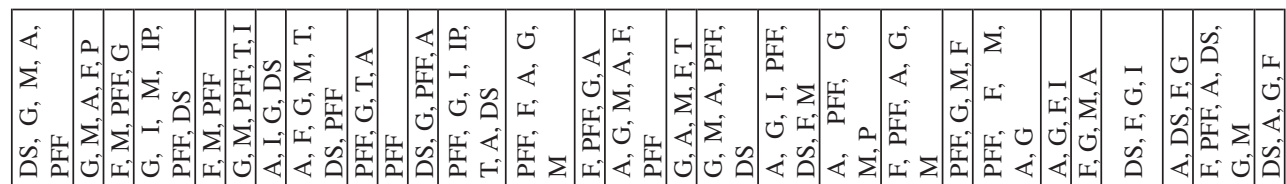

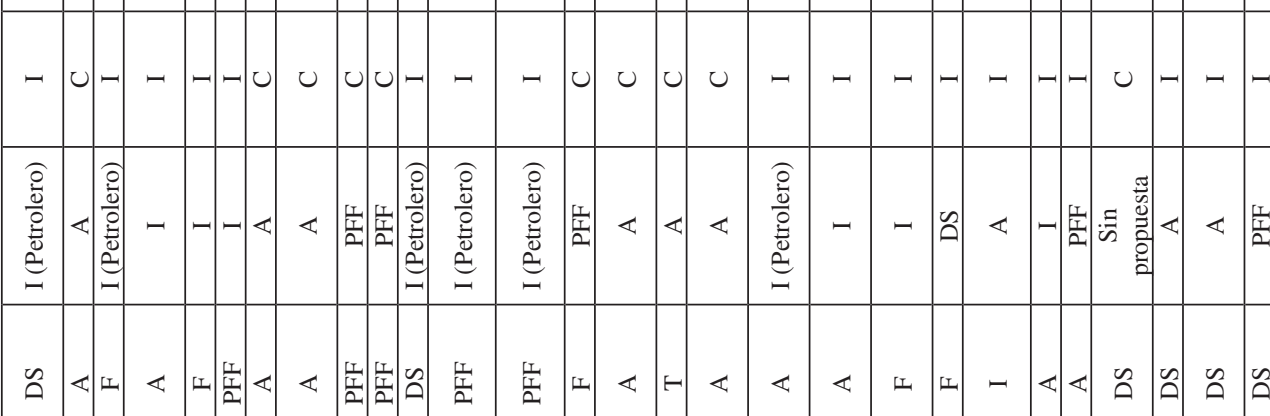

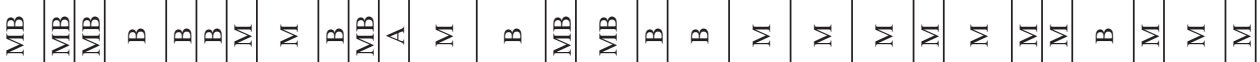

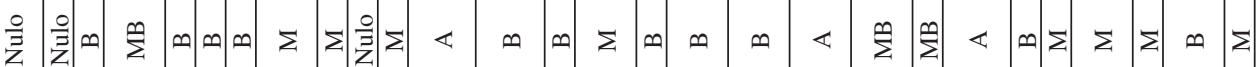

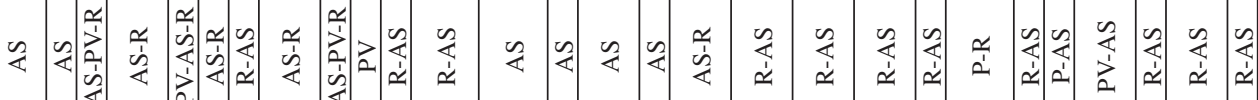
*

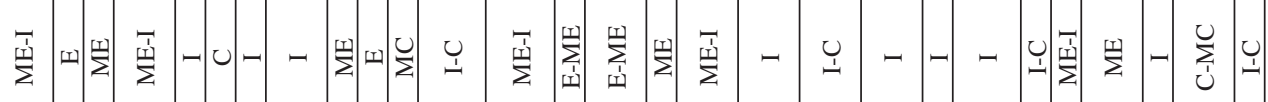

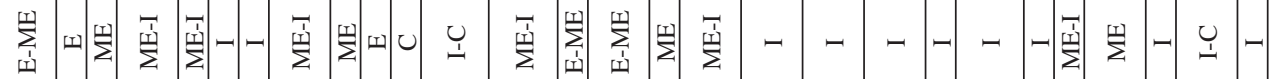

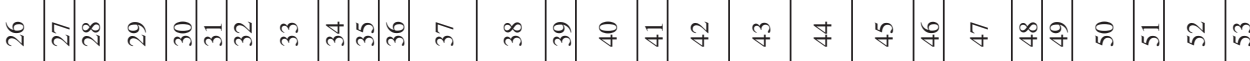




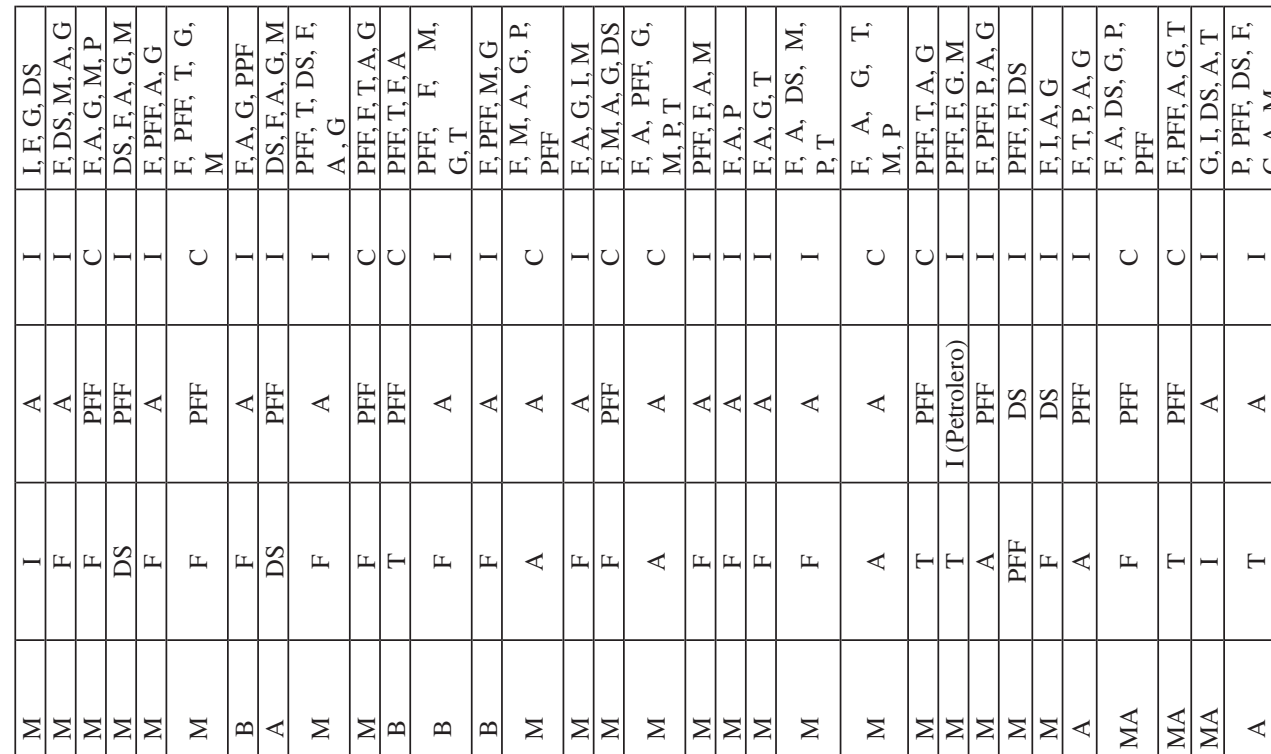

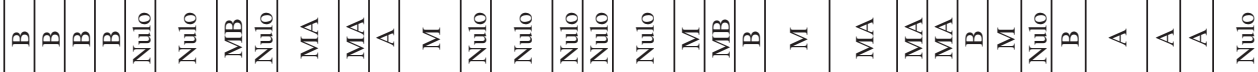

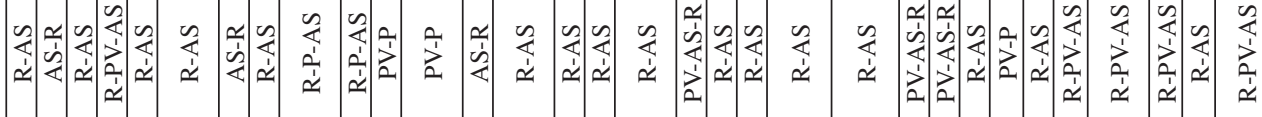

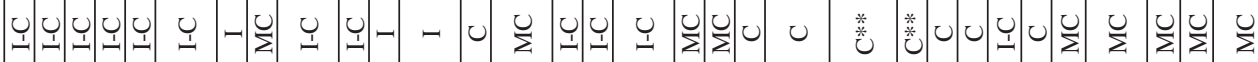
II

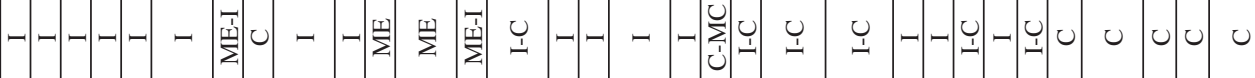

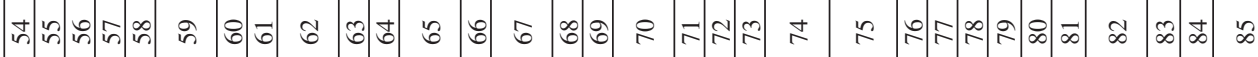




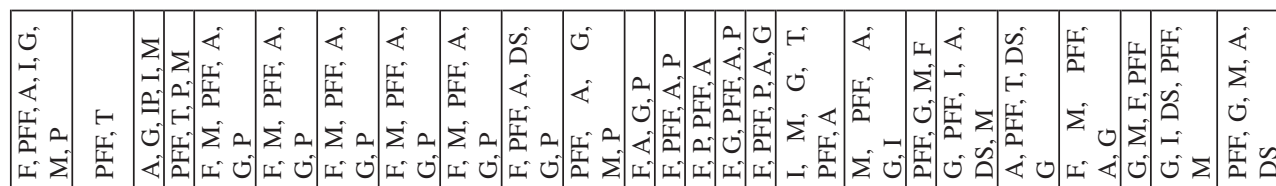

\begin{tabular}{|c|c|c|c|c|c|c|c|c|c|c|c|c|c|c|c|c|c|c|c|c|c|}
\hline & & & & & & & & & & & & & & & & & & & & & \\
\hline- & $u$ & u |- & - & U & - & $u$ & - & - & - & $-1-$ & 0 & -1 & U & - & - & - & $u$ & $u$ & 0 & $u$ & - \\
\hline$\varangle$ & 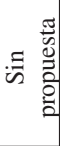 & \begin{tabular}{l|l}
0 \\
0 \\
0 \\
0 \\
0 \\
0 \\
0 \\
0
\end{tabular} & $\ll$ & $\ll$ & $\ll$ & $\varangle$ & $\varangle$ & $\varangle$ & - & $|<|$ & & & 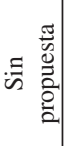 & $\tilde{n}$ & $\varangle$ & $\tilde{\Omega}$ & $\varangle$ & $\ll$ & 䇏 & 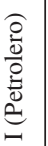 & $\varangle$ \\
\hline- & 空 & $\varangle H$ & $\omega$ & $\varangle$ & 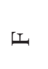 & $\ll$ & $H$ & 山 & L & 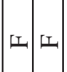 & & 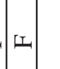 & $H$ & $\frac{\sqrt[T]{L}}{2}$ & 竞 & 崖 & $\varangle$ & $\varangle$ & 咅 & - & 党 \\
\hline$\varangle$ & $\stackrel{m}{\Sigma}$ & $\Sigma \mid \tilde{\Sigma}$ & $\Sigma$ & $\infty$ & $\infty$ & $\stackrel{m}{\Sigma}$ & $\infty$ & $\stackrel{m}{\Sigma}$ & $\infty$ & $\ll \mid \Sigma$ & $\sum \mid \Sigma$ & $\Sigma$ & $\stackrel{n}{\Sigma}$ & $\stackrel{n}{\Sigma}$ & $\Sigma$ & $\tilde{\Sigma}$ & $\infty$ & $\Sigma$ & $\tilde{\Sigma}$ & $\stackrel{n}{\Sigma}$ & $\Sigma$ \\
\hline$\frac{O}{3}$ & $\frac{\bigcirc}{z}$ & $\mathbb{~}$ & $\Sigma$ & $\stackrel{\circ}{\stackrel{\Xi}{z}}$ & $\frac{O}{\bar{z}}$ & $\stackrel{m}{\Sigma}$ & $\Sigma$ & $\sum^{n}$ & $\stackrel{\oplus}{\Sigma}$ & $\infty \frac{0}{3}$ & 을 & $\frac{0}{3}$ & 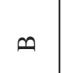 & $\frac{\circ}{\bar{z}}$ & $\infty$ & $\frac{\circ}{\bar{z}}$ & $\stackrel{\circ}{\bar{z}}$ & $\stackrel{\circ}{\frac{3}{z}}$ & 을 & $\frac{\circ}{\bar{z}}$ & $\infty$ \\
\hline
\end{tabular}

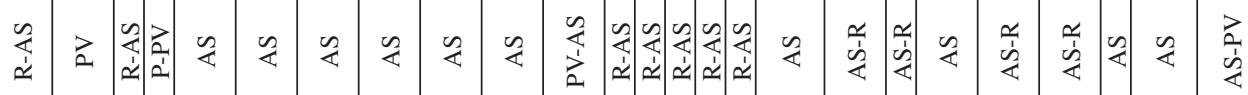

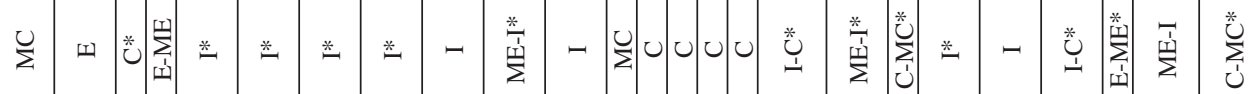

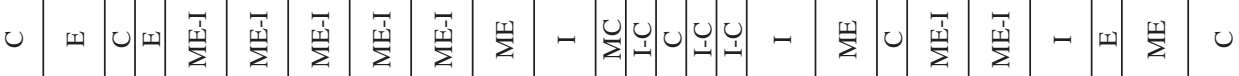

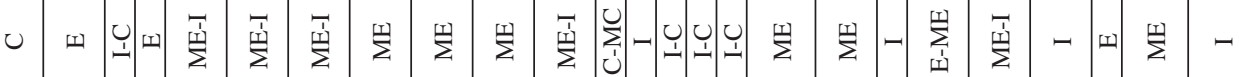

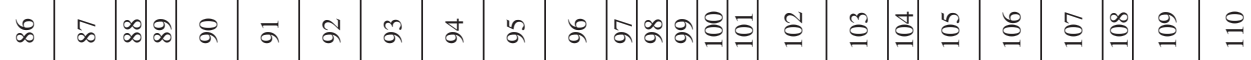




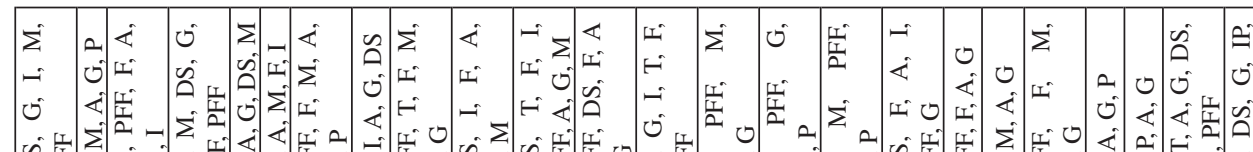

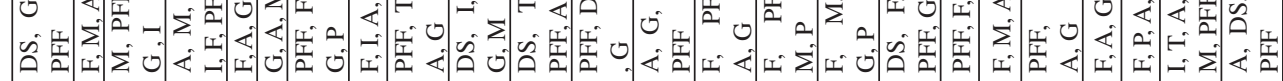

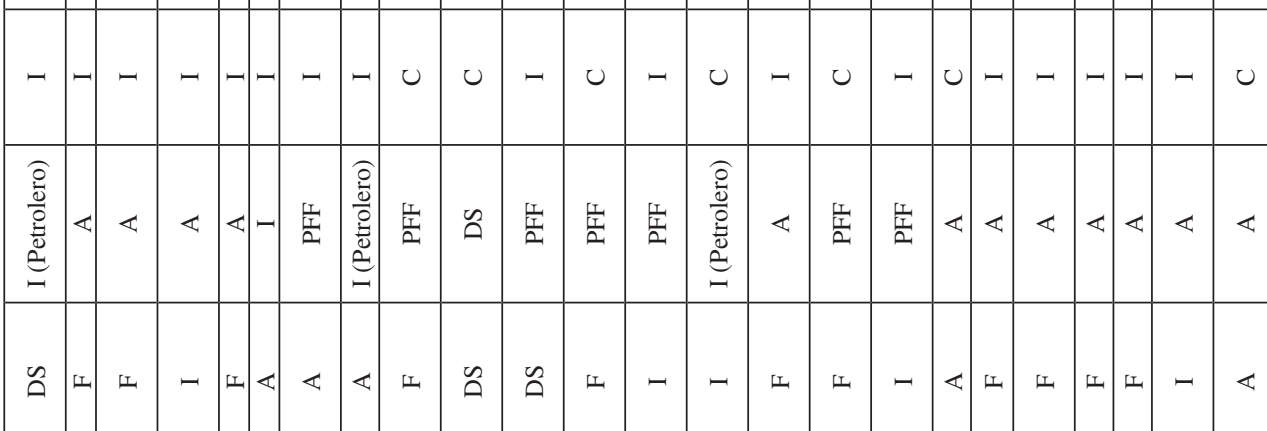

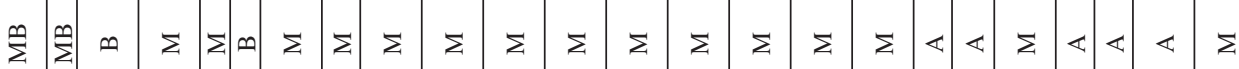

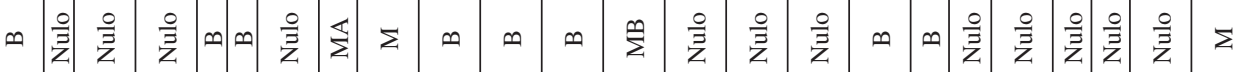

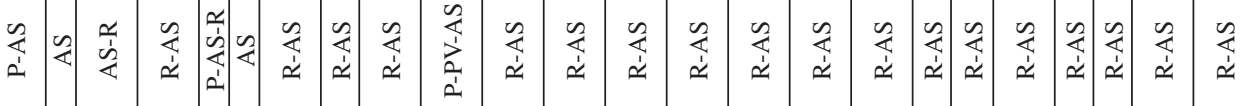

艺

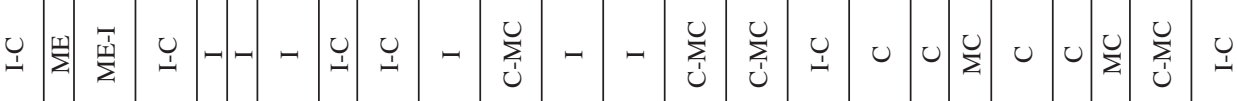

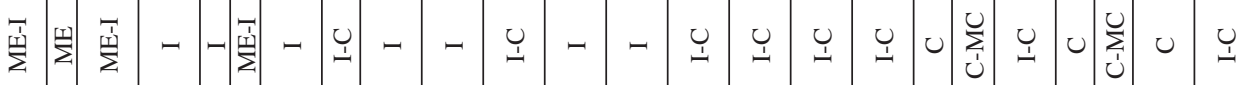

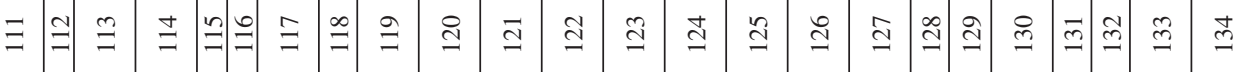




\begin{tabular}{|c|c|c|c|c|c|c|c|c|}
\hline 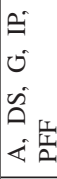 & 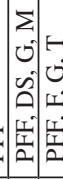 & & 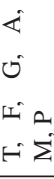 & 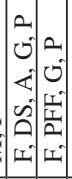 & & 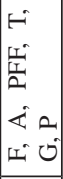 & 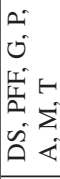 & 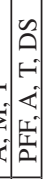 \\
\hline U & u & 0 & - & - & - & - & U & 0 \\
\hline 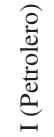 & 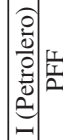 & & $\varangle$ & $\left|\frac{1}{2}\right|<\mid$ & $\varangle$ & $\varangle$ & $\ll$ & $\varangle$ \\
\hline- & $|I| I \mid$ & 4 & $I$ & 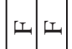 & 工 & $\omega$ & $\varangle$ & $\varangle$ \\
\hline$\Sigma$ & $|\Sigma| \Sigma$ & $\Sigma$ & $\Sigma$ & $<\Sigma$ & $\varangle$ & $\varangle$ & $\varangle$ & $\ll$ \\
\hline$\Sigma$ & $|\Sigma| \Sigma$ & 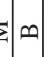 & $\Sigma$ & $\stackrel{\oplus}{\Sigma} \frac{\circ}{\vec{z}}$ & $\infty$ & $\stackrel{\circ}{\Xi}$ & $\infty$ & $\sum \mid$ \\
\hline $\begin{array}{l}\underset{4}{\alpha} \\
\propto\end{array}$ & 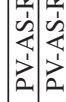 & & $\begin{array}{l}\sum_{1}^{n} \\
\stackrel{1}{n}\end{array}$ & 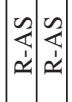 & $\begin{array}{l}\underset{1}{n} \\
\alpha\end{array}$ & 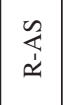 & 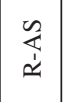 & 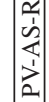 \\
\hline 莌 & ـ - & 羏 & $U$ & 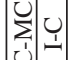 & $\sum$ & $\sum_{\Sigma}$ & $\sum_{\Sigma}^{U}$ & $\sum$ \\
\hline I & - & - & U & $0-1$ & U & U & $u$ & 0 \\
\hline- & & - & 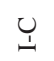 & $0-$ & U & $U$ & U & 0 \\
\hline$\stackrel{m}{2}$ & 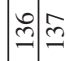 & {$[\stackrel{\infty}{m}$} & ले & 竓 & $\Xi$ & 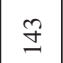 & 过 & a \\
\hline
\end{tabular}

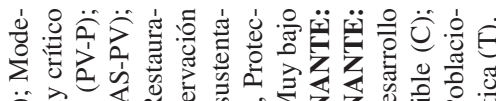

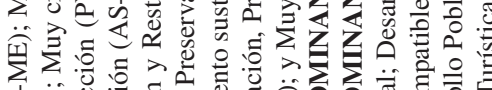

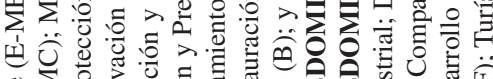

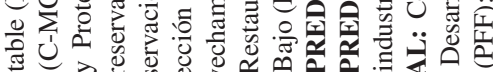

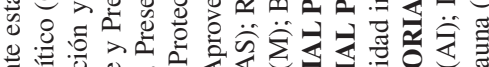

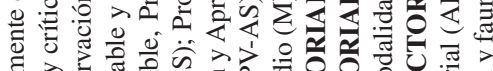

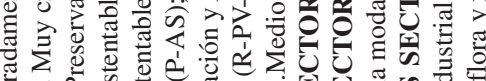

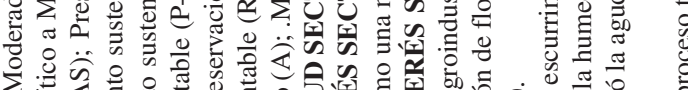

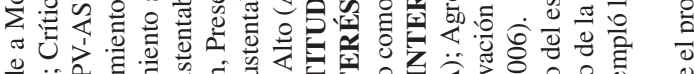

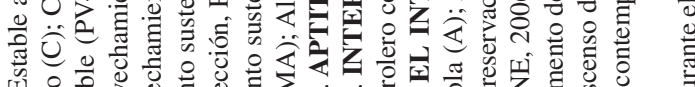

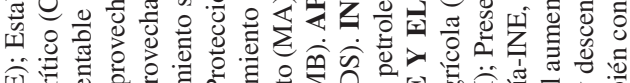

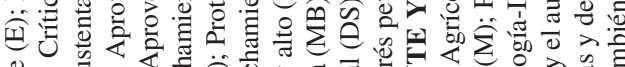

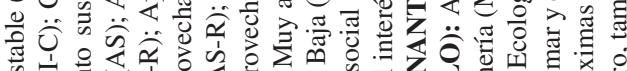

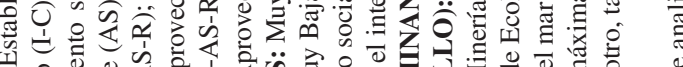

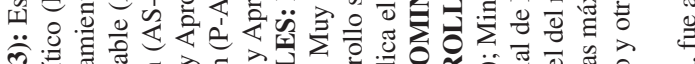

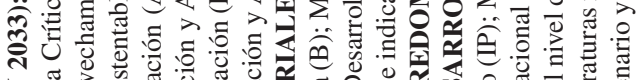

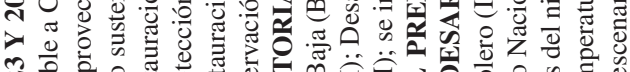

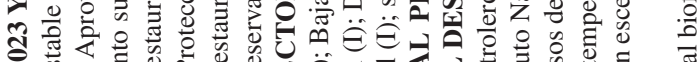
สิ

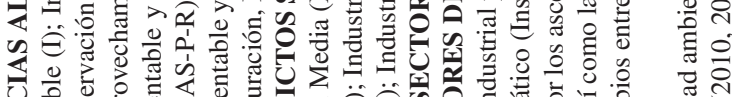

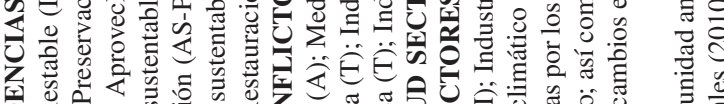

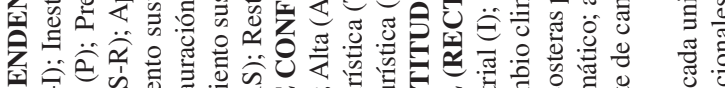

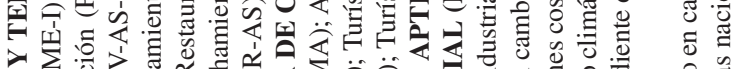

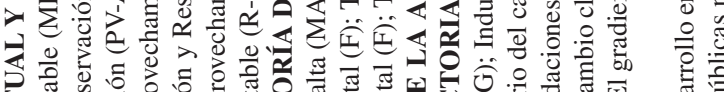

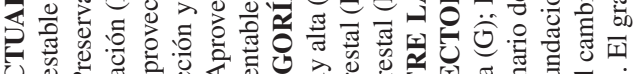

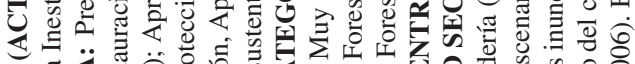

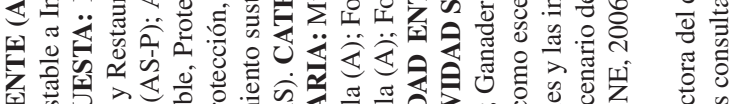

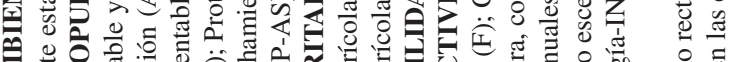

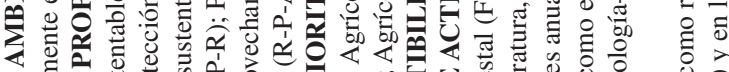

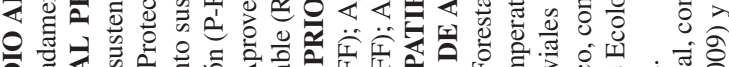

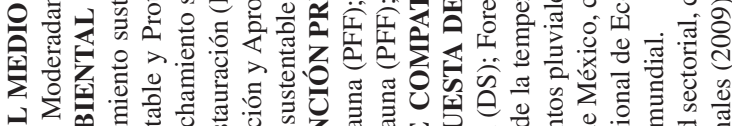

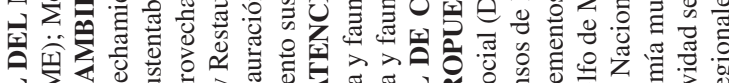

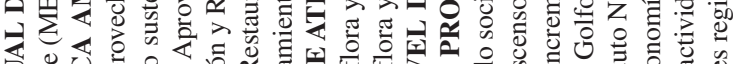

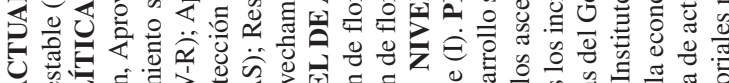

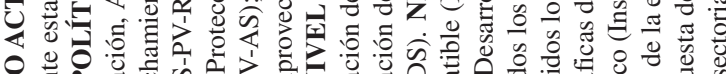

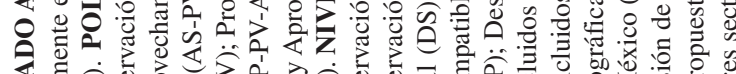

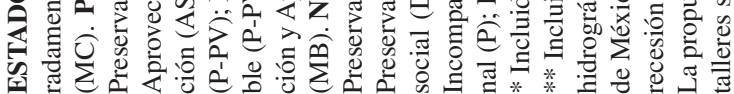


Finalmente, se desarrolló la formulación de la propuesta del MOEGT, con base en los productos de la caracterización y del diagnóstico, el grado de compatibilidad entre la aptitud sectorial y el interés de cada sector, asimilando los escenarios del pronóstico. Las valoraciones finales para cada UAB sobre el estado actual del medio ambiente; sus proyecciones tendenciales a 2012, 2023 y 2033; la política ambiental propuesta; la categoría de conflictos sectoriales; el nivel de atención prioritaria; la aptitud y el interés sectoriales predominantes; el grado de compatibilidad entre ellos; así como la propuesta de actividad sectorial para el desarrollo sustentable, se plasman en la Tabla 3. La implementación del modelo de ocupación sectorial, se rigió por 10 lineamientos ambientales generales en tres grupos propositivos (dirigidos a la sustentabilidad ambiental del territorio, al mejoramiento del sistema social y la infraestructura urbana, y al fortalecimiento de la gestión y la coordinación institucional). Estos lineamientos presentaron 44 estrategias ambientales y 273 acciones de implementación, la gran mayoría en consonancia con el Plan Nacional de Desarrollo 2006-2012.

En el procesamiento de la información para la integración de las variables necesarias para la caracterización, el diagnóstico integrado, el pronóstico y la propuesta de ocupación sectorial, se empleó el SIG Arc Gis, v. 9.3

\section{RESULTADOS Y DISCUSIÓN}

El análisis de los procesos de degradación ambiental biofísica en México reportó 31.5 $\%$ de desertificación por influencia antropogénica (Hernández Cerda, 2008a), $50.2 \%$ del fondo nacional de suelos con algún tipo de degradación (López García, 2008b) y $47.9 \%$ de la cobertura vegetal presenta diferentes manifestaciones de degradación (Sánchez Colón et al., 2008a, 2008b). A partir de la información cartográfica sobre estos procesos degradantes y de otros indicadores sociales y económicos, se pudo establecer el proceso de evaluación medioambiental. Dado que el ordenamiento ecológico posee una profunda observancia hacia el aprovechamiento sustentable, la preservación, protección y restauración del patrimonio natural, la valoración del estado actual del medio ambiente constituyó un acertado enfoque para la conformación del escenario contextual del país, así como de sus proyecciones a 15 y 25 años, es decir a los años 2023 y 2033 (Fig. 3).

Este proceso de identificación y evaluación medioambiental fue esencial para la planeación ecológica del territorio nacional, pues permitió establecer un pronóstico básico de ocupación futura, sobre la base de la proposición del modelo deseable más viable, contando con la instrumentación sectorial de las políticas federales sustentables y siempre de acuerdo con las políticas ambientales requeridas para el territorio nacional (HernándezSantana et al., 2009). Precisamente, esta esencia ambiental del POEGT, determinó el análisis contextual y tendencial bajo la óptica de la evaluación geoecológica del estado actual del medio ambiente, según la metodología de Mateo et al. $(1994,2001)$ y sobre la base de la pérdida de potenciales naturales, la pérdida y degradación de los suelos, la extensión y distribución de los procesos de desertificación y la expresión de distintos indicadores sociales y económicos (Tabla 1). Además, se consideraron los problemas de integridad de la unidad regional (UAB), teniendo en cuenta el grado de desarticulación de la estructura y del funcionamiento de las mismas, que determinan el incumplimiento de sus funciones ambientales. En este sentido, el estado del medio ambiente de las UAB quedó clasificado 
en las categorías siguientes: regiones en estado estable, estable a medianamente estable, medianamente estable, medianamente estable a inestable, inestable, inestable a crítico, crítico, crítico a muy crítico y muy crítico.

Cabe destacar, que a estos efectos, el medio ambiente se consideró como un sistema abierto, de formación histórica, producto de la interacción entre la naturaleza, la sociedad y su economía (Bucek, 1983; Arcia, 1994; Mateo et al., 2001). En general, la evaluación del estado actual (2008) del medio ambiente del territorio nacional mostró que un $47.10 \%$ del país presenta un estado entre inestable y crítico a muy crítico, concentrándose en la región sur-sureste y algunas regiones del centro del país. TERRITORIO NACIONAL PARA CADA ESCENARIO TEMPORAL (2008, 2012, 2023 Y 2033)

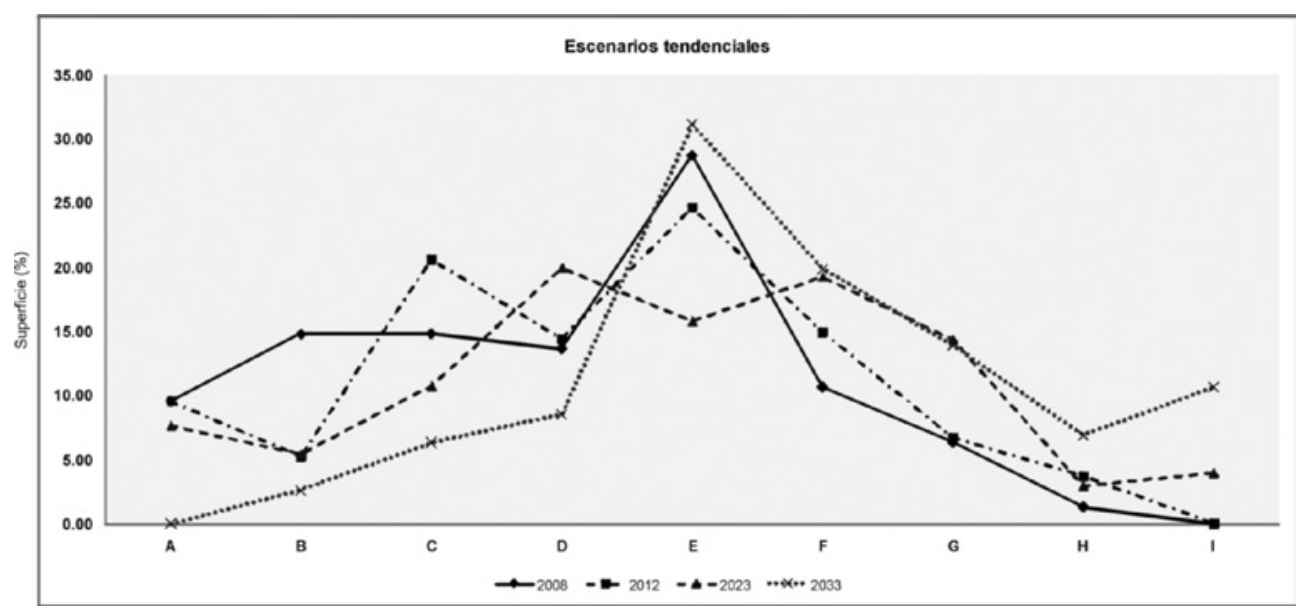

A. Estable, B. Estable a medianamente estable, C. Medianamente estable, D. Medianamente estable a inestable, E. Inestable, F. Inestable a crítico, G. Crítico, H. Crítico a muy crítico, I. Muy crítico. Obsérvese como disminuyen las áreas con categorías de estabilidad relativa y aumentan las inestables, críticas y muy críticas en el escenario del año 2033 con respecto a los años 2008, 2012 y 2023.

Los escenarios tendenciales del estado del medio ambiente al 2023 y 2033 incluyeron, además de las 18 variables (Tabla 1), algunas consideraciones sobre el cambio climático, tales como, los ascensos de la temperatura, la humedad del suelo, los incrementos pluviales anuales y las inundaciones costeras por los ascensos del nivel del mar y el aumento del escurrimiento superficial en las cuencas hidrográficas del Golfo de México, como escenario del cambio climático (INE, 2006). Las evaluaciones y ponderaciones de todas las variables incluyeron el juicio de expertos obtenido en los talleres regionales y sectoriales de consulta y validación, fundamentalmente sobre las tendencias de la degradación del suelo y la vegetación, la desertificación, el crecimiento poblacional, la marginación social y el grado de conflicto y/o sinergia intersectoriales. 
En el escenario del año 2023, el estado medioambiental de las UAB con categoría de estable, estable a medianamente estable y medianamente estable ocuparían el $23.81 \%$ del país, localizándose fundamentalmente en su región norte. Estas categorías se reducen en un $11.63 \%$ con respecto al escenario del 2008. Por otra parte, las categorías de inestable a crítico, crítico, crítico a muy crítico y muy crítico se concentrarían en los estados de Chiapas, Oaxaca, Guerrero, Puebla, Veracruz, Michoacán, Tamaulipas, partes de Jalisco (costa del Pacífico), Yucatán y Quintana Roo, con un $40.48 \%$ del territorio nacional. Para el escenario del año 2033, las categorías de estabilidad ambiental se reducirían hasta el $8.96 \%$ del país, localizándose fundamentalmente en la Península de Baja California y sectores de Coahuila, Chihuahua y Nuevo León; mientras los niveles de criticidad ambiental ocuparían el $51.34 \%$ de las regiones centro y sur-sureste de México (Fig. 3).

Ante estos escenarios de tendencias nacionales, el escenario estratégico o de políticas ambientales contempló los criterios sobre sinergias y conflictos intersectoriales, la degradación biofísica, el nivel de modificación antropogénica, las características del nivel de desarrollo social y económico en cada UAB y el análisis del porcentaje de áreas naturales protegidas federales por UAB y su tipo, para determinar la aplicación o no de la política de preservación y, mediante el análisis de las categorías de manejo de las áreas naturales protegidas, identificar aquellas que requieren una política de protección, dado que poseen un significado para el fomento de servicios ambientales, tales como, uso forestal y recarga de acuíferos. Por supuesto, que dada la diversidad de situaciones ambientales, el escenario estratégico incluyó 18 combinaciones de políticas, a partir de las cuatro básicas contempladas en la LGEEPA de los años 1988 y 2003: aprovechamiento sustentable, preservación, protección y restauración.

Según la LGEEPA (SEMARNAT, 2003), tres principios deben regir la regionalización ecológica, más bien ecólogo-sectorial por su contenido, dado que considera los intereses de preservación de flora y fauna, y protección ambiental, pero además de los restantes sectores de la APF. Esos principios son la política ambiental, el nivel de atención prioritaria y la aptitud sectorial. De acuerdo con las categorías del estado actual de medio ambiente (2008) y el grado de conflictos o sinergias ambientales, se clasificaron cinco niveles de atención prioritaria (muy alta, alta, media, baja y muy baja); las UAB del sur-sureste concentraron una atención prioritaria muy alta y alta, mientras que hacia el norte predominaron UAB de muy baja atención prioritaria, dada la estabilidad medioambiental actual y un bajo grado de conflictos intersectoriales.

Para la estimación de la aptitud sectorial, se registró la presencia o la ausencia de variables o indicadores ambientales, tomados como descriptores y condicionantes para el desarrollo de cada actividad sectorial en específico, siempre cumpliendo el requisito de su representación cartográfica. De acuerdo con la información obtenida en entrevistas con los diversos sectores y en los talleres regionales, se procesaron y ponderaron estadísticamente los atributos ambientales ofrecidos por cada sector para obtener tres niveles de aptitud sectorial: alto, medio y bajo (Ongay y Zorrilla, 2008b). El método utilizado consistió en una evaluación multicriterio, que utiliza la suma ponderada de los valores de cada variable (la escala de evaluación va de 0 a 10), mientras la ponderación se efectuó promediando los coeficientes sugeridos por los expertos con base en el proceso de análisis jerárquico de Saaty (1980). Con esta aplicación, se obtuvieron capas digitales para determinar la aptitud sectorial de doce sectores, aunque de ellas sólo siete se refieren a grados de aptitud, los otros sectores 
expresaron ausencia o presencia del mismo, estado de las actividades normativas sectoriales, como la gestión ambiental, en general, o informativos sectoriales, pero los datos facilitados no constituyeron una plataforma para la evaluación de la aptitud del sector. Otros sectores no están debidamente representados, como consecuencia de la ausencia de información precisa.

Partiendo de este análisis y de la información disponible, se procedió a clasificar los niveles de aptitud sectorial para los sectores de actividad forestal, preservación de flora y fauna, agrícola, industrial, minería, ganadería, turístico, desarrollo poblacional y desarrollo social. Para cada sector, una vez correlacionados sus atributos ambientales, se estableció una jerarquización cualitativa de su aptitud en 10 categorías (alta, alta a media, media a alta, media, media a baja, baja a media, baja, baja a no apto, no apto a baja y no apto), en dependencia de la extensión superficial de sus categorías de aptitud sectorial alta, media y baja. Una vez identificados los niveles de aptitud sectorial, se estimó la aptitud sectorial predominante en cada UAB. Finalmente, la regionalización ecológica (ecológico-sectorial) incluyó 80 regiones, agrupadas según políticas ambientales, niveles de atención prioritaria y las aptitudes sectoriales predominantes en cada UAB. No obstante esa jerarquización, no se excluyeron de la propuesta de ordenamiento ecológico ni de la información cartográfica, a aquellos intereses sectoriales de menor ocupación espacial.

La confrontación de las aptitudes sectoriales por UAB y los intereses sectoriales en cada una de ellas, propició el análisis de compatibilidad entre ambas, como vía para la definición de la propuesta de uso territorial (Tabla 3). De esta manera, fueron determinados 34 tipos de propuestas de uso sectorial en el modelo de ordenamiento ecológico general del territorio (MOEGT), para cada una de las cuales fueron diseñados sus lineamientos, estrategias y acciones ecológicas concretas.

\section{CONCLUSIONES}

Las investigaciones desarrolladas en el POEGT de México tienen un corte analítico e interpretativo sobre el estado actual del patrimonio natural del país; sobre sus niveles de degradación; sobre las áreas que deberían estar sujetas a planes de protección, conservación y restauración; y sobre los espacios geográficos más vulnerables ante el desarrollo de procesos y/o fenómenos naturales peligrosos. Esta información cartográfica, compuesta por 24 mapas, a escala 1:2 000 000, permitió determinar la aptitud sectorial para el desarrollo, como primer paso para la integración del análisis de congruencia de los programas sectoriales, determinándose las áreas de conflictos por concurrencia espacial de sectores, por competencia de recursos y por incongruencia de programas sectoriales.

La aplicación de la metodología del análisis del estado del medio ambiente por unidades ambientales biofísicas, basándose en el análisis espacio-temporal de indicadores de degradación ambiental, de modificación antropogénica y de estado de desarrollo social y económico, y en la influencia de cambios climáticos futuros, refleja escenarios alarmantes para el territorio mexicano, que requieren la inmediata aplicación de políticas ambientales y públicas, que contribuyan a mitigar los efectos degradativos en la calidad ambiental, en la equidad social y en el crecimiento económico, bajo un prisma de planeación territorial adecuada, mejor distribución presupuestaria federal, estatal y municipal, en función de las necesidades nacionales y de los sectores más marginados. Esta metodología permitió «espacializar» los principales 
problemas actuales y evaluar el estado medioambiental, así como proyectar sus tendencias a diferentes plazos temporales.

El análisis del escenario estratégico o de políticas ambientales indica, que de las 18 combinaciones de políticas ambientales para México, las de mayor extensión y necesidad de aplicación inmediata son aprovechamiento sustentable y restauración (26.09 \% del país), aprovechamiento sustentable $(24.76 \%)$, restauración y aprovechamiento sustentable (14.99 $\%)$ y protección, aprovechamiento sustentable y restauración $(6.11 \%)$ y preservación y protección (5.29\%); las restantes son inferiores al $5 \%$ del territorio nacional.

Tanto la regionalización ecológica (ecólogo-sectorial) como el modelo de ordenamiento ecológico general del territorio contemplaron, como enfoque de sustentabilidad, las evaluaciones de la aptitud sectorial predominante o vocación territorial más eficiente, unido al nivel de prioridad de atención del territorio, como un instrumento federal robusto para el desarrollo de actividades sectoriales compatibles con la vocación natural de las UAB y su ajuste en función de la dinámica nacional. La presentación y el debate consensuado del MOEGT en los talleres regionales de expertos y funcionarios de la APF, así como en las consultas públicas nacionales de los años 2010 y 2011, con su necesaria retroalimentación, enriquecieron dicho instrumento gubernamental de óptica estratégica para el derrotero del desarrollo sectorial del país y de salvaguarda de su patrimonio ambiental.

Para próximas actualizaciones del POEGT, es requisito indispensable el desarrollo de proyectos previos con los distintos sectores de la APF, dirigidos a la organización de la información digital sectorial y ambiental, como base para el diseño de sistemas de información geográfica, que garanticen el almacenamiento de información más segura, precisa y oportuna, y amplíen la indispensable cultura cartográfica institucional para la ejecución de proyectos de corte territorial y sobre todo de reorganización sectorial del espacio.

El proceso de elaboración del Programa de Ordenamiento Ecológico General del Territorio (POEGT) tuvo un carácter analítico y sintético multidisciplinario e intersectorial en las condiciones geográfico-ambientales y productivas mexicanas, dirigidos a la sustentabilidad nacional, bajo la óptica de la Administración Pública Federal, siempre como visión estratégica territorial de gran horizonte, en armonía con las escalas de acción estatal y municipal, más locales, tácticas y operativas, pero vitales para la optimización de uso del territorio y la conservación de su medio ambiente.

\section{AGRADECIMIENTOS}

Los autores agradecen a la SEMARNAT y al INE el apoyo presupuestario a los proyectos «Caracterización, diagnóstico y propuesta para el ordenamiento ecológico general del territorio», «Pronóstico del ordenamiento ecológico general del territorio» $\mathrm{y}$ «Asistencia técnica para la consulta pública del POEGT». A su vez, agradecen a Antonio Díaz de León Corral, Araceli Vargas Mena y Amezcua, Santa Castro Miranda, Martín Aguilar Hernández, Francisco Gaytán Gama, Juan Claudio Fonseca Valencia, por la SEMARNAT, y a Eduardo Peters Recagno, Fernando Rosete Verges, Gerardo Negrete Fernández, Leobardo Terpán Acuña, Gustavo Hernández Bortolini, Nora Esquivel Esquivel e Iveth Hernández Montoya, por el Instituto Nacional de Ecología, toda su colaboración y recomendaciones durante el proceso de ejecución de los referidos proyectos. Reconocen la profesionalidad y el desempeño de Arturo Vicente Arreola 
Muñoz, Gontrán Villalobos Sánchez, Rosario Espinoza Martínez, Lina María Erazo Quintero y Tomás Darío Pérez Vega en la facilitación del debate técnico participativo durante la celebración de los talleres regionales de expertos y en las consultas públicas nacionales (2010, 2011). Agradecen a Ailsa Winton la traducción inglesa del resumen ampliado y a los árbitros sus valiosos comentarios y acertadas sugerencias durante el proceso académico de dictaminación.

\section{REFERENCIAS BIBLIOGRÁFICAS}

ARCIA RODRÍGUEZ, M. (1994): «La Geografía del Medio Ambiente». Facultad de Planeación Urbana y Regional. Universidad Autónoma del Estado de México, Toluca, 289 p.

BUCEK, A. (1983): «Problemática de las investigaciones geográficas del medio ambiente». Studia Geographica, No. 86, pp. 17-25.

CASADO IZQUIERDO, J. M. (2008a): «Marginación municipal 2005». En: Caracterización y diagnóstico integrado para el Programa de Ordenamiento Ecológico General del Territorio (Hernández-Santana, J. R., coord.). SEMARNAT, México, D. F., 1 hoja a escala 1:2 000000.

CASADO IZQUIERDO, J. M. (2008b): «Distribución y dinámica de la población por localidad, 1990-2005. En: Caracterización y diagnóstico integrado para el Programa de Ordenamiento Ecológico General del Territorio (Hernández-Santana, J. R., coord.). SEMARNAT, México, D. F., 1 hoja a escala 1:2 000000.

CASADO IZQUIERDO, J. M. (2008c): «Tasa de dependencia económica municipal». En: Pronóstico para el Programa de Ordenamiento Ecológico General del Territorio (Hernández-Santana, J. R., coord.). SEMARNAT, México, D. F., t. III, pp. 23-24.

CASADO IZQUIERDO, J. M. (2008d): «Porcentaje de trabajadores por actividades renumeradas por municipios (población económicamente activa ocupada con ingresos mayores a dos salarios mínimos, 2000)». En: Pronóstico para el Programa de Ordenamiento Ecológico General del Territorio (Hernández-Santana, J. R., coord.). SEMARNAT, México, D. F., t. III, pp. 24-25.

CONABIO (2008): «Conocimiento actual de la biodiversidad». En: Capital natural de México. CONABIO, México, D. F., 620 p.

http://www.biodiversidad.gob.mx/pais/pdf/CapNatMex/Vol\%20I/I00PrefacioGuia.pdf

CUESTA AGUILAR, M. J. (2006): «Ordenación del territorio, medio ambiente y globalización: reflexiones desde la geografía regional al nuevo contexto socio-económico». Boletín de la A.G.E., No. 42, pp. 255-269.

DELGADO VIÑAS, C. (2006): «Ordenación del territorio y desarrollo sostenible en áreas de montaña: diagnóstico y propuestas para la integración productiva y territorial de los Montes de Pas (Cantabria), Boletín de la A.G.E., No. 42, pp. 53-70.

DE LUCIO, J. V., ATAURI, J. A., SASTRE, P., MARTÍNEZ C. (2003): «Conectividad y redes de espacios naturales protegidos: del modelo teórico a la visión práctica de la gestión». En: Conectividad ambiental: las áreas protegidas en la cuenca mediterránea (García Mora, M. R., coord.). Junta de Andalucía, pp. 29-54.

DIARIO OFICIAL DE LA FEDERACIÓN (1988): «Ley General del Equilibrio Ecológico y la Protección al Ambiente». México, 28 de enero de 1988. México, D. F., 103 p.

FERNÁNDEZ MUÑOZ, S. (2008): «Participación pública, gobierno del territorio y paisaje en la comunidad de Madrid». Boletín de la A.G.E., No. 46, pp. 97-119. 
FRANCO, A. (2009): «Ordenamiento territorial en Panamá». Rev. Planeamiento territorial y urbanismo iberoamericana, No. 6, Ciudad de Panamá.

GRABOW, S. H., HILLIKER, M., MOSKAL, J. (2006): «Comprehensive planning and citizen participation». Madison, WI: UW-Extension Publication G3810.

GURRUTXAGA SAN VICENTE, M., PEIO LOZANO V. (2009): «La integración de la conectividad ecológica en los instrumentos de ordenación y planificación territorial: una revisión». Boletín de la A.G.E., No. 49, pp. 45-66.

HERNÁNDEZ CERDA, M. E. (2008a): «Proceso de desertificación». En: Caracterización y diagnóstico integrado para el Programa de Ordenamiento Ecológico General del Territorio (Hernández-Santana, J. R., coord.). SEMARNAT, México, D. F., t. I-II, pp. 70-89.

HERNÁNDEZ CERDA, M. E. (2008b): «Intensidad de las sequías». En: Caracterización y diagnóstico integrado para el Programa de Ordenamiento Ecológico General del Territorio (Hernández-Santana, J. R., coord.). SEMARNAT, México, D. F., t. I-II, pp. 147-159.

HERNÁNDEZ-SANTANA, J. R., BOLLO-MANENT, M., MÉNDEZ-LINARES, A. P., LÓPEZ-MIGUEL, C. (2009): «Pronóstico para el Ordenamiento Ecológico General del Territorio (Hernández-Santana, J. R., coord.)». INE, México, D. F., 208 p.

INE (2006): «Comité Intersecretarial de Cambio Climático, México. Tercera Comunicación Nacional ante la Convención Marco de las Naciones Unidas sobre el Cambio Climático». Instituto Nacional de Ecología, México, D. F., 212 p.

INEGI (2008): «Anuario Estadístico de los Estados Unidos Mexicanos, 2008. México, D. F., $679 \mathrm{p}$.

INEGI (2010): «Resultados Preliminares de los Estados Unidos Mexicanos. Censo de Población y Vivienda 2010». México, D. F.

http://www.inegi.org.mx/sistemas/TabuladosBasicos/preliminares2010.aspx

INTERNATIONAL MONETARY FUND (IMF) (2009): «Nominal 2009 GDP for the world and the European Union». World economic outlook database.

JUÁREZ GUTIÉRREZ, M. DEL C., GARCÍA DE LEÓN, A. (2007a): «Índice medio de educación». En: Nuevo Atlas Nacional de México (Coll-Hurtado, A., coord.), Instituto de Geografía, UNAM, México, D. F., p. S XII 2.

JUÁREZ GUTIÉRREZ, M. DEL C., GARCÍA DE LEÓN, A. (2007b): «Índice medio de salud». En: Nuevo Atlas Nacional de México (Coll-Hurtado, A., coord.), Instituto de Geografía, UNAM, México, D. F., p. S XII 3.

JUÁREZ GUTIÉRREZ, M. DEL C., A. (2007c): «Tenencia y Hacinamiento de la vivienda». En: Nuevo Atlas Nacional de México (Coll-Hurtado, A., coord.), Instituto de Geografía, UNAM, México, D. F., p. S XI 6.

JUÁREZ GUTIÉRREZ, M. DEL C., A. (2007d): «Consolidación de la vivienda». En: Nuevo Atlas Nacional de México (Coll-Hurtado, A., coord.), Instituto de Geografía, UNAM, México, D. F., p. S XI 5.

LÓPEZ BLANCO, J. (2007): «Mapa de regiones ambientales biofísicas de México, a escala 1:4 000 000». En: Nuevo Atlas Nacional de México (Coll-Hurtado, A., coord.), Instituto de Geografía, UNAM, México, D. F.

LÓPEZ BLANCO, J. (2008): «Regionalización ambiental (biofísica) nacional». En: Caracterización y diagnóstico integrado para el Programa de Ordenamiento Ecológico General del Territorio (Hernández-Santana, J. R., coord.). SEMARNAT, México, D. F., t. I-II, pp. 319-353. 
LÓPEZ GARCÍA, J. (2008a): «Tipos de degradación de suelos». En: Caracterización y diagnóstico integrado para el Programa de Ordenamiento Ecológico General del Territorio (Hernández-Santana, J. R., coord.). SEMARNAT, México, D. F., t. I-II, pp. 29-57.

LÓPEZ GARCÍA, J. (2008b): «Áreas con suelos degradados». En: Caracterización y diagnóstico integrado para el Programa de Ordenamiento Ecológico General del Territorio. SEMARNAT, México, D. F., t. I-II, pp. 290-301.

MATEO, J., DA SILVA, V., BRITO CAVALGANTE A. P. (1994): «Analise da paisage como base para estrategio de Organizacao Geoambiental: Corumbatai cspl Colectario 004 Planeamiento Ambiental». Universidad de Sao Paulo, pp. 57-105.

MATEO RODRÍGUEZ, J. M., ORTIZ-PÉREZ, M, A. (2001): «La degradación de los paisajes como concepción teórico- metodológica». Nueva Época, No. 1, Instituto de Geografía, UNAM, México, D. F., 40 p.

MORALES, J., COLL-HURTADO, A. (2007): «Capitalización de la industria, 2003». En: Nuevo Atlas Nacional de México (Coll-Hurtado, A., coord.), Instituto de Geografía, UNAM, México, D. F., p. E VIII 9.

ONGAY DELHUMEAU, E., ZORRILLA, M. (2008a): «El ordenamiento ecológico general del territorio y las políticas públicas de la Administración Pública Federal». SEMARNAT, México, D. F., 76 p.

ONGAY DELHUMEAU, E., ZORRILLA M. (2008b): «Aptitud sectorial». En: Formulación de la propuesta del Programa de Ordenamiento Ecológico General del Territorio (Hernández-Santana, J. R., coord.). SEMARNAT, México, D. F., t. IV, pp. 5-58.

ROMERO GONZÁLEZ, J., FARINÓS DASI, J. (eds.) (2004): «Ordenación del territorio y desarrollo territorial». Gijón, Ediciones Trea, 352 p.

SAATY, T. L. (1980): «The analytic Hierarchy Process: Planning, Priority setting, Resource allocation. Pittsburgh, Pa: RWS Publications, 281 p.

SÁNCHEZ COLÓN, S., TREJO VÁZQUEZ, I., CRUZ LEYVA, I. A. (2008a): «Grados de deterioro de la vegetación». En: Caracterización y diagnóstico integrado para el Programa de Ordenamiento Ecológico General del Territorio (Hernández-Santana, J. R., coord.). SEMARNAT, México, D. F., t. I-II, pp. 58-69.

SÁNCHEZ COLÓN, S., TREJO VÁZQUEZ, I., CRUZ LEYVA, I. A. (2008b): «Áreas con degradación de la cobertura vegetal». En: Caracterización y diagnóstico integrado para el Programa de Ordenamiento Ecológico General del Territorio (Hernández-Santana, J. R., coord.). SEMARNAT, México, D. F., t. I-II, pp. 306- 318.

SEMARNAP, ANALÍTICA CONSULTORES Y CONSULTORES INTERNACIONALES, S. C. (2000): «Prospectiva para el modelo de ordenamiento ecológico territorial». México, D. F., 3 t.

SEMARNAT (2003): «Reglamento de la Ley General del Equilibrio Ecológico y la Protección al Ambiente en materia de ordenamiento ecológico». Diario Oficial de la Federación, 8 de agosto de 2003. México, D. F., pp. 39-58.

SEMARNAT (2009): «Estadísticas del agua en México». México, D. F., p.98.

SEMARNAT (2012): «Programa de Ordenamiento Ecológico General del Territorio». México, D. F., 90 p.

ZOIDO, F. (1998): «Geografía y Ordenación del Territorio». Didáctica de las Ciencias Sociales, 16, pp. 19-31. 
